world

trade

institute

\title{
Testing for Convergence in \\ Carbon Dioxide Emissions using a \\ Bayesian Robust Structural Model
}

Octavio Fernández-Amador, Doris A. Oberdabernig and Patrick Tomberger

WTI Working Paper No. 01/2017

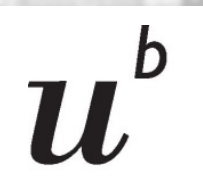




\title{
Testing for Convergence in Carbon Dioxide Emissions using a Bayesian Robust Structural Model *
}

\author{
Octavio Fernández-Amador ${ }^{\dagger}$ \\ Doris A. Oberdabernig $\ddagger$ \\ Patrick Tomberger ${ }^{\S}$
}

\begin{abstract}
We address international convergence in Carbon Dioxide emissions per capita and per value added derived from emission inventories based on production and consumption patterns. We propose a Bayesian structural model that accounts for heteroscedasticity, endogeneity between emissions and economic growth, and tests for the existence of group-specific convergence via shrinkage priors. We find evidence for countryspecific conditional convergence in all emission inventories, implying a half-life of 2.7-3.1 years for production-based emissions and 3.6-4.7 years for consumption-based emissions. When testing for global convergence without allowing for individual-specific convergence paths, the half-life of $\mathrm{CO}_{2}$ per capita increases to $15-26$ years, whereas emission intensities show a half-life of $44-45$ years. Our results highlight the current incompatibility between emission targets and economic growth and the need for faster diffusion of green technologies. Moreover, there is no evidence for specific convergence dynamics in the European Union, the OECD, or the countries that ratified the Kyoto Protocol. The institutional frameworks implemented in industrialized countries did not induce faster convergence among developed economies.
\end{abstract}

Keywords: $\mathrm{CO}_{2}$ emissions, production-based inventories, carbon footprint, convergence test, half-life.

JEL-codes: F18, F64, O44, Q54, Q56.

* The authors acknowledge support of the NCCR Trade Regulation, grant No. 51NF40-151576, University of Bern.

$\dagger$ Corresponding author: World Trade Institute, University of Bern, Hallerstrasse 6, CH-3012 Bern (Switzerland). E-mail address: octavio.fernandez@wti.org.

¥ World Trade Institute, University of Bern, Hallerstrasse 6, CH-3012 Bern (Switzerland). E-mail address: doris.oberdabernig@wti.org.

$\S$ World Trade Institute, University of Bern, Hallerstrasse 6, CH-3012 Bern (Switzerland). E-mail address: patrick.tomberger@wti.org. 


\section{Introduction}

Global warming and its consequences are at the center of current policy debate on the sustainability of economic development. The Paris Agreement stipulates holding the global average temperature below $2^{\circ} \mathrm{C}$ above pre-industrial levels to bring climate change under control $;^{1}$ for this to happen, the 194 countries that signed the agreement are compelled to reach the global peak of greenhouse gas (GHG) emissions as soon as possible (Paris Agreement, Art. 2 and 4). The underlying question is how to make economic growth compatible with limited or decreased pollution, particularly GHG emissions.

The Environmental Kuznets Curve (EKC) predicts that pollution increases with rising income per capita and falls after a peak in emissions has been reached. However, the existence of a turning point in GHG emissions after which emissions start to decrease with economic growth has not been unanimously confirmed by empirical research. Especially for Carbon Dioxide $\left(\mathrm{CO}_{2}\right)$ emissions the existence of such peak has often been rejected. ${ }^{2}$ Against this background, it is important to know whether global carbon emissions will eventually reach a limit; only then the growth rate of atmospheric concentrations of $\mathrm{CO}_{2}$ will stabilize.

The patterns of convergence of $\mathrm{CO}_{2}$ emissions per capita towards a certain emission level and the height of this level have important implications for the design of the international regulatory framework. Reliable information on whether the steady state of emissions is global or country-specific and on how long it will take for countries to reach this steady state can strengthen the ongoing policy debate. Related to this, the convergence dynamics of carbon emissions derived from both national production and consumption activities should be better understood when revising environmental responsibility, as they characterize the path of emissions associated with further economic development in a globalized context. Increasingly fragmented value chains allow the geographical location of production stages to differ from the place of final consumption. A mere focus on territorial-based emissions neglects the importance of trade in intermediates and carbon leakage, i.e. the shift of highly pollutant industries from countries with stringent environmental regulation to countries with less strict regulation (e.g. Aichele and Felbermayr, 2015; Babiker, 2005; Fernández-Amador et al., 2016).

1 See Knutti and Fischer (2015) for a critical analysis of the $2^{\circ} \mathrm{C}$ target.

2 Empirical studies that investigate the existence of an $\mathrm{EKC}$ in $\mathrm{CO}_{2}$ emissions usually fail to find such a relationship in samples covering a large group of countries (see e.g. Stern, 2004, and Stern, 2017, for exhaustive surveys, or Fernández-Amador et al., 2017, for a survey of empirical applications). Aslanidis and Iranzo (2009) and Fernández-Amador et al. (2017) provided evidence that the income elasticity of $\mathrm{CO}_{2}$ emissions decreases as income per capita rises above a threshold level though emissions continue growing, what challenges the sustainability of economic growth. 
In addition, it is relevant to understand to what extent the dynamics of international convergence of emissions per capita is driven by convergence in carbon efficiency worldwide. The adoption of more environmentally friendly technologies will lower carbon intensities, which is particularly relevant for developing countries, as they need to combine remarkable economic growth targets with emission reduction goals. ${ }^{3}$ If international technology transfers occur and emerging economies adopt greener production methods, the global production network will eventually become more sustainable, and $\mathrm{CO}_{2}$ emissions per value added will converge across countries. This will in turn promote convergence in emissions per capita.

The assessment of convergence in $\mathrm{CO}_{2}$ emissions has received considerable attention in the empirical literature. ${ }^{4}$ Most studies tested for convergence in $\mathrm{CO}_{2}$ per capita across different groups of countries, but their results remain broadly inconclusive. ${ }^{5}$ By contrast, a smaller number of studies investigated convergence in carbon efficiency, pointing invariably towards the existence of convergence across countries. ${ }^{6}$ However, all these studies focused on production-based emissions, while cross-country convergence in $\mathrm{CO}_{2}$ embodied in consumption has not yet been investigated. ${ }^{7}$

We evaluate international convergence in $\mathrm{CO}_{2}$ emissions per capita and per value added derived from national production- and consumption-based inventories worldwide. We put forward a Bayesian test of $\beta$-convergence that is based on the theoretical models by Brock and Taylor (2010) and Ordás Criado et al. (2011). Our model also allows for potential group-specific dynamics of convergence using Bayesian shrinkage priors. Our convergence test is robust to heteroscedasticity and accounts for potential endogeneity between the growth rates of emissions and GDP per capita by means of instrumental variables (IV) estimation.

3 The Paris Agreement recognizes the need to support developing countries in order to facilitate the effective implementation of the objectives identified in the Agreement (Paris Agreement, Art. 2).

4 See Pettersson et al. (2014) and Stern (2017) for comprehensive surveys of the literature on convergence in pollution emissions.

5 The findings of the literature range from evidence for convergence (Strazicich and List, 2003; Nguyen, 2005; Ezcurra, 2007; Romero-Ávila, 2008; Lee et al., 2008; Westerlund and Basher, 2008; Lee and Chang, 2009; Brock and Taylor, 2010; Jobert et al., 2010; Huang and Meng, 2013; Yavuz and Yilanci, 2013; Anjum et al., 2014; Hao et al., 2015; Wu et al., 2016; Zhao et al., 2015) over the existence of convergence clubs (Nguyen, 2005; Aldy, 2006; Lee and Chang, 2008; Panopoulou and Pantelidis, 2009; Barassi et al., 2011; Ordás Criado and Grether, 2011; Camarero et al., 2013; Herrerias, 2013; Wang et al., 2014; Burnett, 2016) to no evidence for convergence (Aldy, 2007; Barassi et al., 2008; Nourry, 2009).

6 See Anjum et al. (2014), Camarero et al. (2013) and Panopoulou and Pantelidis (2009).

7 Aldy (2007) investigates convergence of $\mathrm{CO}_{2}$ emissions across US states. This is the only study so far that also covers consumption inventories. The author did not find evidence for convergence for either $\mathrm{CO}_{2}$ production or for $\mathrm{CO}_{2}$ consumption per capita. In contrast to Aldy, our study covers economies at different development states, thus being the first one to evaluate global convergence patterns in $\mathrm{CO}_{2}$ consumption. 
Our contribution is twofold. First, we assess international convergence in production- and consumption-based carbon emissions for the first time by using a comprehensive dataset on comparable $\mathrm{CO}_{2}$ emission inventories published, and recently updated, by FernándezAmador et al. (2016). The dataset covers 178 economies (grouped in 66 countries and 12 composite regions) and extends over 17 years after the Kyoto Protocol ratification. A period marked by the implementation of environmental policies against climate change in developed countries. The focus on both inventories allows to account for the increasing detachment between $\mathrm{CO}_{2}$ per capita generated by production activities and $\mathrm{CO}_{2}$ embodied in final consumption in a period of rapidly expanding global production networks, which permit cross-border sourcing of carbon in final consumption. In addition, we analyze $\mathrm{CO}_{2}$ emissions per value added (carbon intensity or efficiency) and draw conclusions on whether the detected patterns are driven by efficiency effects. While $\mathrm{CO}_{2}$ per capita offers important insights on convergence stemming from the expansion of production or consumption in a country, convergence in $\mathrm{CO}_{2}$ intensity provides information on whether countries that use more pollutant production methods eventually catch up with environmentally more efficient economies.

Second, our structural model presents some interesting features. It uses a Bayesian stochastic search variable selection prior (SSVS, George and McCulloch, 1993) to test for the existence of group-specific convergence dynamics. The groups comprise the European Union (EU), the OECD, and the countries that ratified Annex I of the Kyoto Protocol. The model is robust to cross-sectional heteroscedasticity - it is based on a scale mixture of multivariate normals, where the hyperparameter governing the distribution of the individual-specific variances is estimated endogenously. Furthermore, it formulates a flexible Cholesky-prior to instrument potentially endogenous regressors. This prior was proposed by Lopes and Polson (2014) in the framework of normal distributions, and, to the best of our knowledge, this is the first time this prior is applied in the context of scale mixtures of multivariate normals. ${ }^{8}$

Our results point to the existence of country-specific conditional convergence in all four emission inventories. The speed of convergence implies a half-life of 2.7-3.1 years for production inventories and 3.6-4.7 years for $\mathrm{CO}_{2}$ consumption. Convergence towards global steady states, though conditioned on the political and economic structures, is much slower, implying a half-life of 15 and 26 years for emissions production and, respectively, consumption per capita and 44-45 years for emission intensities. Moreover, we do not find

\footnotetext{
8 Salois and Balcombe (2015) proposed a related model in the context of cross-section, where $t$-distributed errors in an IV-model are represented by weighted the errors of normals. Their modelization shares with ours the use of scale mixture of normals representation, though the authors do not perform Choleskyrotation of the system to represent it as a recursive system of equations but condition the weighted errors on each other and use a Wishart prior for the variance-covariance matrix.
} 
support for the existence of group-specific convergence dynamics for countries belonging to the OECD, the EU, or the Annex I of the Kyoto Protocol. These findings evince the ineffectiveness of environmental policies implemented in developed economies and pose doubts on the feasibility of an effective global action against climate change.

The next section reviews the literature on convergence. Section 3 describes the data. In section 4, we explain the specification of the convergence test. Section 5 presents the results and section 6 concludes.

\section{Literature review}

Convergence tests received considerable attention in the empirical literature evaluating the predictions of the Solow (1956) growth model. Early studies tested whether countries starting out from low income levels experienced higher subsequent growth rates, either conditional on or unconditional of control variables ( $\beta$-convergence). ${ }^{9}$ Later studies suggested that $\beta$-convergence could be driven by regression to the mean (see Friedman, 1992; Quah, 1993) and tested whether the dispersion of income across countries was narrowing over time ( $\sigma$-convergence) ${ }^{10}$ Yet, Sala-i-Martin (1996) pointed out the merits of $\beta$-convergence for providing insights into growth dynamics. Although $\beta$-convergence is not sufficient for $\sigma$-convergence, it is a necessary condition (Sala-i-Martin, 1996; Young et al., 2008) and provides valuable information whenever alternative tests for convergence cannot be applied. ${ }^{11}$

Besides cross-sectional convergence tests, also time-series approaches have been developed. Several authors investigated stochastic convergence of income levels via unit root testing, that is, whether income shocks are of permanent or temporary nature. ${ }^{12}$ While these approaches became increasingly popular as more data became available over time, Bernard and Durlauf (1996) pointed out that they are grounded on the assumption that

9 Earlier studies focused on unconditional convergence, while more recent studies tested for conditional convergence, i.e. convergence after allowing for heterogeneity across countries by accounting for additional determinants of economic growth. While unconditional convergence was often found for OECD countries, it was generally rejected for samples including non-OECD countries. If countries converge to different steady states, unconditional convergence models might result in biased coefficient estimates as the model used for estimation is miss-specified (see Barro and Sala-i Martin, 2004). See for example Baumol (1986); Barro (1991); Barro and Sala-i Martin (1992); Mankiw et al. (1992); Barro and Sala-i Martin (2004).

10 See e.g. Barro and Sala-i Martin (1992); Quah (1993); Sala-i-Martin (1996); Young et al. (2008). Phillips and Sul (2007b) developed a test for identifying club-convergence groups, which corresponds to a test of conditional $\sigma$-convergence (see Phillips and Sul, 2007b). Phillips and Sul (2007a) provided a short empirical application of the test in the context of economic growth convergence.

11 See e.g. Ravallion (2003) who applies $\beta$-convergence tests to international income inequality.

12 See e.g. Carlino and Mills (1993); Quah (1993); Bernard and Durlauf (1996); Evans and Karras (1996). 
the economies in the sample are near their long-run equilibria. In this sense, the use of time-series tests may be invalid if the data are driven by transition dynamics. ${ }^{13}$

Similar to the Solow model for economic growth, there are theoretical models that predict convergence of pollution emission levels across countries over time (e.g. Brock and Taylor, 2010; Ordás Criado et al., 2011). Like the Solow model, these boil down econometrically to an equation of conditional $\beta$-convergence.

Empirical studies on convergence in $\mathrm{CO}_{2}$ per capita derived from production activities led to inconclusive findings. For OECD countries, Strazicich and List (2003), Romero-Ávila (2008), Lee et al. (2008), Lee and Chang (2009), Jobert et al. (2010), and Yavuz and Yilanci (2013) found evidence for convergence. Lee and Chang (2008) and Barassi et al. (2011) reported convergence only for a subgroup of countries, and Barassi et al. (2008) did not detect evidence for convergence. ${ }^{14}$

A growing number of studies included developing countries in their samples. Ezcurra (2007), Westerlund and Basher (2008), Brock and Taylor (2010), and Anjum et al. (2014) provided evidence for convergence across countries of different income status. Panopoulou and Pantelidis (2009), Ordás Criado and Grether (2011), and Herrerias (2013) detected several convergence clubs, ${ }^{15}$ and Nguyen (2005) and Aldy (2006) found convergence only in sub-groups or clubs of developed economies. Nourry (2009) failed to detect evidence for cross-country convergence.

Some authors focused on convergence across regions in China and the US. For China, Huang and Meng (2013) detected overall convergence and Wu et al. (2016) found evidence for club convergence. For the US, Burnett (2016) found a club of 26 converging states, while convergence for the US as a whole was rejected. While all these studies focused on $\mathrm{CO}_{2}$ production inventories, Aldy (2007) additionally assessed consumption of $\mathrm{CO}_{2}$ per capita in the US states, but did not find convergence in either measure.

The heterogeneous findings of the literature on $\mathrm{CO}_{2}$ convergence are in line with the inconclusive evidence for the existence of an environmental Kuznets curve (EKC). The EKC hypothesis suggests that as national income levels rise, pollution first increases with income, but after a certain level of income has been reached this mechanism is reversed. ${ }^{16}$ If income levels are positively correlated with $\mathrm{CO}_{2}$ emissions, the existence of an EKC

\footnotetext{
13 See also Panopoulou and Pantelidis (2009), Jobert et al. (2010) and Ordás Criado and Grether (2011) for surveys on $\beta$-, $\sigma$ - and stochastic convergence.

14 Studies for OECD countries focused mainly on stochastic and $\beta$-convergence. For more details on the concept of convergence used by the respective studies, see Table A.1 in the Appendix.

15 Panopoulou and Pantelidis (2009) and Herrerias (2013) applied the Phillips and Sul (2007b) test for convergence clubs. Ordás Criado and Grether (2011) found evidence for income-specific and regional convergence clubs especially for the sub-period 1980-2000.

16 See Dasgupta et al. (2002), Kaika and Zervas (2010), and Stern (2004, 2017) for reviews, and FernándezAmador et al. (2017) for a summary of the most recent evidence.
} 
relationship would ultimately lead to emission convergence (Stern, 2017). However, even though empirical studies find a positive relationship between economic growth and $\mathrm{CO}_{2}$ emissions, the evidence favoring an EKC-type relationship is restricted to time-series or panel studies covering OECD economies. ${ }^{17}$

Improvements in carbon efficiencies (i.e. $\mathrm{CO}_{2}$ per value added) are an important requirement for reaching the turning point postulated by the EKC. High-income countries generally are more carbon efficient than less developed economies (Fernández-Amador et al., 2016). This can be explained by their stronger preferences for a cleaner environment, better access to cleaner technology and potential for carbon leakage. Carbon leakage will impede convergence in carbon emission intensities, as firms with larger emission intensity might relocate to countries with less stringent environmental regulation. However, if the rapid increase in international trade induces transfers of green technology to less developed countries, their carbon efficiency could improve more rapidly (Grossman and Helpman, 1995), which would contribute to convergence in carbon intensities. Thus, although most studies focused on $\mathrm{CO}_{2}$ emissions per capita, evaluating convergence in carbon intensities provides additional insights in the convergence patterns across countries. ${ }^{18}$

Among the existing studies on convergence in the intensity of $\mathrm{CO}_{2}$ emissions from production activities, Camarero et al. (2013) identified four convergence clubs among 22 OECD countries using the test for club-convergences developed by Phillips and Sul (2007b). Anjum et al. (2014) and Panopoulou and Pantelidis (2009) provided evidence for convergence in a panel of 136 and 128 countries, respectively. Focusing on Chinese regions, the results of Hao et al. (2015) and Zhao et al. (2015) suggested convergence of emission intensity, while Wang et al. (2014) found evidence for club convergence. ${ }^{19}$

\section{Data}

$\mathrm{CO}_{2}$ emissions per capita and per value added derived from production and consumption inventories are available from the emissions database constructed by Fernández-Amador et al. (2016). Following Fernández-Amador et al. (2016), we define carbon intensities as

\footnotetext{
17 Schmalensee et al. (1998) is an exception, finding support for an inverse-U relationship using nonparametric techniques. More recently, Aslanidis and Iranzo (2009) and Fernández-Amador et al. (2017) found that the income-elasticity of $\mathrm{CO}_{2}$ emissions decreases slightly after income per capita passes a certain threshold, such that relative decoupling increases with economic growth, though there is no evidence of absolute decoupling and an EKC relationship. Fernández-Amador et al. (2017) also provided evidence for a similar pattern in $\mathrm{CO}_{2}$ consumption-based inventories.

18 Anjum et al. (2014) reported that the negative correlation between initial emission and subsequent emission growth is stronger for $\mathrm{CO}_{2}$ intensity than for $\mathrm{CO}_{2}$ per capita.

19 All of these studies define $\mathrm{CO}_{2}$ intensity as $\mathrm{CO}_{2}$ per GDP. In our analysis we refer to $\mathrm{CO}_{2}$ intensity as $\mathrm{CO}_{2}$ per value added.
} 
carbon per value added rather than per GDP. For production inventories, value added is computed as value added embodied in production, whereas for consumption-based inventories, it is calculated as value added embodied in consumption. Therefore, emission inventories and value added are measured at the same stage of the supply chain. The dataset consists of a balanced panel of national production- and consumption-based carbon dioxide emission inventories from fossil fuel combustion covering 66 countries and 12 composite regions (encompassing a total of 178 economies) over the years 1997, 2001, 2004, 2007, 2011 and 2014 (468 observations). ${ }^{20}$ It relies on input-output, trade and energy data of several releases of the Global Trade Analysis Project (GTAP) database.

To test for the presence of $\beta$-convergence, we compute the growth rates of the four emission inventories, which we consecutively use as dependent variables in the empirical analysis. Since the data-points are unequally spaced in time (3 to 4 periods), we calculate the average growth rate of emissions between years $t-s$ and $t$, where $s$ is the number of periods between two observations (see Ravallion, 2003, for a similar methodology). ${ }^{21}$ The resulting average growth rates allow to evaluate convergence in the large- $\mathrm{N}$, small- $\mathrm{T}$ panel dataset, for which time-series methods cannot be used. ${ }^{22}$ Furthermore, by using average growth rates, we avoid to capture short-term fluctuations in emissions that could result in an upward bias of the estimates of the convergence speed (see Ordás Criado et al., 2011).

Our baseline control variables are derived from the theoretical model by Ordás Criado et al. (2011). They comprise the lagged level of $\mathrm{CO}_{2}$ emissions, which should capture potential convergence forces, the growth rate of purchasing-power parity (ppp) adjusted real GDP per capita over the period considered, which should capture the scale effect of economic growth on emissions, and the lagged level of ppp-adjusted GDP per capita, as a proxy for capital per efficient labor (see Ordás Criado et al., 2011, for details). GDP per capita is sourced from the World Development Indicators (WDI) dataset.

To limit potential omitted variable bias (see Barro and Sala-i Martin, 2004), we add a large set of additional control variables capturing economic, structural and institutional

20 A description of the countries included in the composite regions is available in Fernández-Amador et al. (2016). The dataset has been recently extended by the authors to cover the year 2014 .

21 This corresponds to calculating average yearly growth rates. For a similar method see Ravallion (2003), who accounts for the unequal spacing in time between measures of income inequality for large-N, small$\mathrm{T}$ panel data by regressing the difference in inequality between time $t$ and the initial period $t_{1}$ on a constant and initial inequality in time $t_{1}$, both multiplied by a time-trend $(t-1)$. In contrast to Ravallion's data, our panel is balanced in the sense that for every individual we observe all variables in the same points in time. Thus, we can also exploit the variation of the data across time and use initial emissions in year $t-s$ instead of in year $t_{1}$ as a regressor.

22 Bernard and Durlauf (1996) pointed out that the power of time-series tests may be weak when the dynamics do not occur near the steady state. In this sense, time-series approaches to test for stochastic convergence may not be particularly suitable in our context, since data on $\mathrm{CO}_{2}$ emissions covering a global sample of countries are very likely to be driven by transition dynamics rather than being near the steady state. 
characteristics of the individuals in the sample, and include individual-, and time-dummies (see Table A.2 in the Appendix for details on the variables definitions and data sources). We derive trade flows as a share of GDP as well as value added shares of different sectors of the economy (agriculture, energy, light manufacturing, heavy manufacturing, textiles, water services, construction, trade and transport, and remaining services) from the GTAP database. $^{23}$ Data on population density, the share of fossil fuels and nuclear energy in total electricity production, and rents from fossil fuel production as a share of GDP are available from the WDI database. A democracy index, which may channel citizens' preferences for a cleaner environment, is sourced from the Polity IV database (see Farzin and Bond, 2006). Finally, in order to investigate group-specific convergence patterns, we generate dummy variables for members of the EU, OECD, and Annex I of the Kyoto Protocol.

\section{Econometric model}

We develop a Bayesian test for $\beta$-convergence as an extension of the model proposed by Ordás Criado et al. (2011). ${ }^{24}$ The model specification is a dynamic panel that enables to test for the existence of specific convergence groups, accounts for endogeneity of the regressors, and allows for cross-sectional heteroscedasticity of the error terms.

Let $E_{i t}$ be, alternatively, the natural logarithm of $\mathrm{CO}_{2}$ emissions per capita or per value added in country $i$ at time $t$, where $i \subseteq[1, \ldots, N]$ and $t \subseteq[1, \ldots, T]$, and let $G_{i, t, s}=$ $\left(E_{i, t}-E_{i, t-s}\right) / s$ be the average growth rate of $E_{i}$ over the period $t-s$ and $t$. The test is

23 Detailed information on the sector aggregation from the original GTAP sectoral disaggregation is available from the authors upon request.

24 Ordás Criado et al. (2011) tested for convergence in sulfur oxides and nitrogen oxides. Their theoretical model assumes optimal control of pollution emissions at the national level, making it particularly suited for applications to local air pollutants. Nevertheless, the structure of the empirical model they specify is compatible with the green Solow model by Brock and Taylor (2010), which the authors applied to $\mathrm{CO}_{2}$ emissions. Ordás Criado et al. (2011) regressed the average growth rates of emissions over the period $t-5$ to $t$ on the level of emissions at the initial period of the growth rate $(t-5)$, the growth rate of GDP over $t-5$ and $t$, GDP in $t-5$, and time- and individual-dummies by OLS and a non-parametric model. The authors also addressed endogeneity between emissions and GDP by instrumenting GDP and its growth rate with their lagged values (following Barro and Sala-i Martin 1992). Brock and Taylor (2010) developed a theoretical model that also predicts conditional $\beta$-convergence, which the authors applied to $\mathrm{CO}_{2}$ emissions. Although Brock and Taylor's model is applicable to global pollutants, in our empirical approach we follow Ordás Criado et al. (2011) since their empirical analysis makes use of the panel structure of the data and accounts for the potential endogeneity of GDP per capita. 
defined by the following recursive structural model with instrumental equation:

$$
\begin{aligned}
G_{i, t, s}= & \beta E_{i, t-s}+\pi_{0} g_{i, t, s}+\pi_{1} Y_{i, t-s}+\sum_{r}\left[\lambda_{r} z_{r, i, t-s}\right]+ \\
& +\delta_{t}+\alpha_{i}+\sum_{j}\left[\beta_{j} d_{j} E_{i, t-s}\right]+\epsilon_{1, i t} \\
g_{i, t, s}= & \alpha_{i v}+\beta_{i v} L\left(g_{i, t, s}\right)+\epsilon_{2, i t} \\
\left(\epsilon_{1, i t}, \epsilon_{2, i t}\right) \sim & N\left(0, \Sigma \omega_{i}\right)
\end{aligned}
$$

The (average) growth rate of emissions $\left(G_{i, t, s}\right)$ over $t-s$ and $t$ depends on the logarithm of the level of emissions in country $i$ at period $t-s\left(E_{i, t-s}\right)$, the (average) growth rate of real GDP per capita over the period $t-s$ and $t\left(g_{i, t, s}\right)$, the logarithm of real GDP per capita of country $i$ in $t-s\left(Y_{i, t-s}\right)$, a set of control variables as described in the data section $\left(z_{j, i, t-s}\right)$, time-effects $\left(\delta_{t}\right)$, and individual-dummies $\left(\alpha_{i}\right)$. The $d_{j}$ 's are dummy variables for group membership in the EU, OECD, and Annex I of the Kyoto Protocol. The parameter associated with $E_{i, t-s}$ is the parameter of interest; in particular, $\beta<0$ provides evidence for conditional $\beta$-convergence.

The relationship between the growth rate of emissions and the growth rate of GDP per capita is potentially endogenous. Thus, we follow Barro and Sala-i Martin (1992) and instrument the growth rate of GDP per capita with its growth rate in the previous period, denoted $L\left(g_{i, t, s}\right)$, as described in equation $(2)$, where $L(\cdot)$ is the lag operator. ${ }^{25}$

The priors for the parameters in (1)-(2) are collected in the following set of equations:

$$
\begin{aligned}
\beta & \sim N(0, \tau) \\
\pi_{0}, \pi_{1}, \lambda_{r}, \delta_{t}, \alpha_{i v}, \beta_{i v} & \sim N(0, \phi) \\
\alpha_{i} & \sim N(0, \psi) \\
\beta_{j} & \sim\left(1-\gamma_{j}\right) N\left(0, \kappa_{0}^{2}\right)+\gamma_{j} N\left(0, \kappa_{1}^{2}\right) \\
\gamma_{j} & \sim \operatorname{Bernoulli}(p) \\
\omega_{i}^{-1} & \sim \operatorname{Gamma}(\nu / 2, \nu / 2)
\end{aligned}
$$

The prior of $\beta$ follows a normal distribution with zero mean and precision $\tau$, where $\tau=$ $(2 / 3)^{2}$, such that the case for unit root in the original dynamic model of emissions is not ruled out. ${ }^{26}$ The priors of the parameters $\pi_{0}, \pi_{1}, \lambda_{r}, \delta_{t}, \alpha_{i v}$, and $\beta_{i v}$ follow a normal

$\overline{25}$ For the first period in our sample, 1997-2001, we use the average growth rate for a period of the same length, 1993-1997, as instrument.

${ }^{26}$ In the original dynamic model of emissions, once we undo the average growth rate, the relevant parameter for the case of regular sampling every period is $(1+\beta)$. The precision elicited ensures that the 
distribution with zero mean and precision $\phi=0.2 .{ }^{27}$ We estimate the individual-effects using the dummy variables approach, where $\alpha_{i}$ is normally distributed with precision $\psi=0.5 .^{28}$ An intercept of the model can be retrieved as $\bar{\alpha}=\frac{1}{N} \sum_{i}^{N} \alpha_{i}$.

Equations (7)-(8) characterize a hierarchical SSVS shrinkage prior (George and McCulloch, 1993) that grants flexibility for the data to discriminate among models including group-specific convergence dynamics (for EU, OECD, and Annex I membership). Each group-specific prior on $\beta_{j}$ is modeled as a mixture of two normals with different precisions $\kappa_{0}^{2}$ and $\kappa_{1}^{2} \cdot \kappa_{0}^{2}>\kappa_{1}^{2}$ so that when $\gamma_{j}=0, \beta_{j}$ is restricted to be estimated around 0 , whereas when $\gamma_{j}=1, \beta_{j}$ remains unrestricted. We set $\kappa_{0}^{2}=10$ and $\kappa_{1}^{2}=1$. To reflect the absence of prior beliefs about the existence of specific group convergence we set $p=0.5$.

The prior elicited in equation (9) defines the distribution of the variances of the individualspecific error terms $\left(e_{1, i t}, e_{2, i t}\right)$. Each individual-specific variance parameter takes the form $\Sigma \omega_{i}$, such that the model exhibits cross-sectional heteroscedasticity. The equation is defined in terms of precisions (inverse of the variances). The gamma prior for the precisions is equivalent to a $\chi^{2}(\nu) / \nu$ and characterizes the model as a scale mixture of normals, where the weights are individual-specific. ${ }^{29}$

The hyperparameter $\nu$ is estimated endogenously with prior

$$
\begin{aligned}
\nu & =\lfloor u\rfloor \\
u & \sim \operatorname{Exp}(1 / \lambda), u \in(3,60),
\end{aligned}
$$

where the function $\lfloor\cdot\rfloor$ rounds the values of $u$ to the nearest integer. Exp in equation (11) stands for an exponential distribution where the rate parameter $\lambda$ is set to 25 , such that

\footnotetext{
hypothesis of unit root in our autoregressive model with explanatory variables is not an extreme event in our prior for $\beta$.

27 The precision is defined as the inverse of the variance. A precision of 0.2 implies a variance of 5 .

28 Note that the precision of the individual-dummies is larger than the precision of the rest of the parameters. A uniform prior on the individual fixed effects would lead to improper posterior distributions for the parameters of interest, while very diffuse priors would lead to very slow convergence of the MCMC algorithm used for inference (see e.g. Lancaster, 2008, Ch. 7).

29 Scale mixture of normals with the weights specified as in (9) are equivalent to a $t$-student distribution (see e.g. Andrews and Mallows, 1974; West, 1987; Ding, 2016). The degrees of freedom of the $t$-student are equal to the hyperparameter governing the distribution of the weights $\omega_{i}, \nu$. With growing $\nu$ the distribution converges to a normal distribution, as less probability mass is concentrated at the tails of the distribution. The prior for the weights in the scale mixture of normals, $\omega_{i}$, together with the prior for the components of the variance matrix $\Sigma$ that we will define below, imply a form of crosssectional heteroscedasticity of the gamma type (Andrews and Mallows, 1974; Geweke, 1993; Koop, 2003, Ch. 6; Lancaster, 2008, Ch. 3). There are two main advantages of modeling the problem in terms of scale mixture of normals instead of as a $t$-student distribution. The first one is that the type of heteroscedasticity, cross-sectional in our case, can be explicitly stated. The second is that it is less computational demanding for the numerical algorithm to estimate the posterior distributions of the parameters.
} 
the density function of $\nu$ is centered at a mean of 25, giving substantial prior weight both to fat-tailed error distributions $(\nu<10)$ and error distributions which are effectively Normal $(\nu>40)$. The estimation of $\nu$ renders the specification in (1)-(3) rather flexible. Small values of $\nu$ will yield heteroscedasticity-robust parameter estimates, while as $\nu$ increases the errors' distribution will approach normality (homoscedasticity). We truncate the prior for $\nu$ such that it is contained in the interval $[3,60] .^{30}$

In order to complete the prior for the covariance matrix in (3), we propose a Choleskybased prior for $\Sigma$. Lopes and Polson (2014) have shown the better performance of this type of prior compared to the more widely used approach of specifying an inverted Wishart prior for $\Sigma$ for IV-models in the context of normal-distributed errors. ${ }^{31}$ More specifically, the components of the error vector are modeled based on the recursive conditional regressions arising from the Cholesky decomposition of $\Sigma=A D A^{\prime}$, such that $D=\operatorname{diag}\left(\Sigma_{1 \mid 2}, \Sigma_{22}\right)$ and $A$ is an upper triangular matrix with ones in the main diagonal and upper triangular component $a_{12}=\Sigma_{12} / \Sigma_{22}$. However, the specific modelization of heteroscedasticity by means of scale mixture of normals requires taking into consideration the effect of the Cholesky-rotation in the individual-specific term of the variance (see Ding, 2016). ${ }^{32}$ Thus, equation (3) can be re-written in recursive conditional form, using the specification of the conditionals of a multivariate scale mixture of normals.

$$
\begin{aligned}
\epsilon_{1 \mid 2, i t} & \sim N\left(a_{12} \epsilon_{2, i t}, \Sigma_{1 \mid 2} \omega_{1 \mid 2, i}\right) \\
\epsilon_{2, i t} & \sim N\left(0, \Sigma_{22} \omega_{2, i}\right)
\end{aligned}
$$

where $\Sigma_{11}=\Sigma_{1 \mid 2}+\Sigma_{12}^{2} / \Sigma_{22}$. We must specify priors for $\Sigma_{22}$, the conditional variance $\Sigma_{1 \mid 2}$, the parameter $a_{12}$, which calibrates the strength of the correlation between $\epsilon_{1, i t}$ and $\epsilon_{2, i t}$,

${ }^{30}$ We regard the priors for the parameters of interest $\left(\beta, \pi_{0}, \pi_{1}, \lambda_{j}, \delta_{t}, \alpha_{i v}, \beta_{i v}, \alpha_{i}, \beta_{j}\right)$ as informative. Geweke (1993) shows that under informative (normal) priors for the slopes, both the first and the second moments of the slopes exist. When the priors of the slopes are uninformative, $\nu>2$ ensures existence of the first moments, while $\nu>4$ ensures existence of the second moments. Thus, the truncation defined contains roughly $80 \%$ of the density around the mean of the prior, while ensuring existence of first moments even in the case of noninformative priors for the parameters of interest.

31 We explain the derivation of the IV-prior in terms of covariance matrices because this is common in the literature, though the specification of the priors is in terms of precisions, as carried out in the software. Alternatively, we could use an inverted Wishart prior for $\Sigma, \Sigma \sim I W\left(v_{0}, \Sigma_{0}\right)$, with parameters $v_{0}$ and $\Sigma_{0}$. Priors for covariance matrices and variances have usually been addressed by means of inverted Wishart and inverted Gamma distributions, respectively, while Wishart or Gamma distributions have been used as priors for precision matrices and precisions. Wishart priors have been extensively used in the framework of Bayesian instrumental variable models under normal-distributed errors (see e.g. Kleibergen and Zivot, 2003; Lancaster, 2008, Ch. 8; Rossi, 2005).

32 Ding (2016) used the representation of a multivariate $t$-student distribution as a scale mixture of multivariate normals to derive the conditional distribution of the multivariate $t$-student, which can be represented by the conditional normal distribution times the conditional distribution of the weights. 
as well as the weights in the instrumental, $\omega_{2, i}$, and in the main equation conditional on the instrumental equation, $\omega_{1 \mid 2, i}$. We assign $\Sigma_{22}^{-1}$ and $\Sigma_{1 \mid 2}^{-1}$ a gamma prior with shape and scale parameters $a, b=0.001$ so that we remain uninformative about the precision of the model. $a_{12}$ follows a normal prior centered at zero and with precision $\tau=0.2$. Finally, the priors for $\omega_{2, i}^{-1}$ and $\omega_{1 \mid 2, i}^{-1}$ also follow a gamma distribution with hyperparameter $\nu$, where $d_{m}=\epsilon_{1 i}^{\prime} \Sigma_{11}^{-1} \epsilon_{1 i}$ is the square Mahalanobis distance in the instrumental equation, on which the main equation is conditioned.

$$
\begin{aligned}
\Sigma_{22}^{-1}, \Sigma_{1 \mid 2}^{-1} & \sim \Gamma(a, b) \\
a_{12} & \sim N(0, \tau) \\
\omega_{2, i}^{-1} & \sim \Gamma(\nu / 2, \nu / 2) \\
\omega_{1 \mid 2, i}^{-1} & \sim \Gamma\left((\nu+1) / 2,\left(\nu+d_{m}\right) / 2\right),
\end{aligned}
$$

The relationship between the location and the rate parameters of the gamma priors in (16) and (17) deserves special attention: The location parameter of $\omega_{1 \mid 2, i}^{-1}$ has increased by $1 / 2$ as compared to the location parameter governing $\omega_{2, i}^{-1}$, what reduces the heavytailedness of the (conditional) main equation. The rate parameter in $(17),\left(\nu+d_{m}\right) / 2$, will be larger in comparison with (16), since $d_{m}$ is typically larger than one, what increases the dispersion of the distribution of individuals' variances in the (conditional) main equation. That is, the more extreme the values of the endogenous variable are, the more dispersive is the conditional distribution of the explained variable in the (conditional) main equation.

A Markov Chain Monte Carlo (MCMC) algorithm is used to carry out Bayesian inference. Gibbs-sampling can be applied to all priors specified, including the SSVS prior, equations (7)-(8), the individual-specific weights and the Cholesky-based priors for covariance of the error terms, equations (14)-(17), and the degrees of freedom parameter, equations (10)-(11). ${ }^{33}$ The vector of parameters to estimate is $P=\left(\beta, \pi_{0}, \pi_{1}, \lambda_{r}, \delta_{t}, \alpha_{i}, \alpha_{i v}, \beta_{i v}, \beta_{j}, \gamma_{j}, \nu, \omega_{2, i}^{-1}, \omega_{1 \mid 2, i}^{-1}, \Sigma_{22}^{-1}, \Sigma_{1 \mid 2}^{-1}, a_{12}\right)$. We implement three Markov chains from which, after a burn-in of $7.5 \cdot 10^{5}$ draws, we retain a posterior sample of $7.5 \cdot 10^{5}$ draws each. ${ }^{34}$ We apply a thinning of 3 , ending up with a mixed posterior sample of $7.5 \cdot 10^{5}$ draws. We average across the posterior sample to calculate the posterior means, standard errors and quantiles of the coefficients, and the posterior inclusion probabilities (PIP) of the coefficients associated with specific group convergence. The PIPs of

\footnotetext{
33 See George and McCulloch (1993) for details on the Gibbs sampler for the SSVS prior, and Lopes and Polson (2014) for the details of the Gibbs sampling for IV-estimation in the context of the normal distribution.

34 That was sufficient for the chains to show mixing and the estimates of the coefficients to convergence to their ergodic distribution.
} 
the coefficients for group convergence show the posterior probability of observing specific dynamics associated with those groups.

The model proposed is a dynamic panel model. Nickell (1981) showed that incidental parameters yield inconsistent OLS or Maximum Likelihood (ML) estimates in dynamic panels with short time dimension. The phenomenon is a consequence of having a limited number of observations from which each incidental (individual-specific) parameter is estimated, which in turn contaminates the estimation of the common parameters and, in particular, of the dynamic (autoregressive) parameter. ${ }^{35}$ The literature has proposed alternative estimators with the aim to correct Nickell (1981) bias such as IV estimators, generalized method of moments (GMM) estimators, analytical corrections for the least squares dummy variable (LSDV) estimator, and bias-corrected estimators based on iterative bootstrapping (see Everaert and Pozzi, 2007, for a review of these estimators). Maddala and $\mathrm{Hu}$ (1996) and Hsiao et al. (1999) showed that the Bayesian approach performs fairly well in the context of dynamic panels when $T$ is small, in comparison with some classical estimators.

Our posterior inference is based on the mean of the mixed posterior sample resulting from the Gibbs sample after thinning. In addition, it is based on informative priors. Therefore, we expect that our posterior estimates do not suffer from considerable bias. ${ }^{36}$ A simulation exercise under homoscedasticity and heteroscedasticity confirmed that the Bayesian estimator resulted in substantial bias reduction. The performance of the Bayesian estimator was comparable to, and sometimes outperformed, the performance of differenceGMM (Arellano and Bond, 1991), system-GMM (Blundell and Bond, 1998), an extension of Kiviet's (1995) bias corrected estimator (see Bruno, 2005), and De Vos et al.'s (2015) bootstrap-based bias correction. ${ }^{37}$

Finally, as a robustness check, we also estimate an alternative (homoscedastic) model where the priors in equations (3), (12), and (13) are replaced, respectively, by

35 The concept of incidental parameter and the problem of limited information to estimate incidental parameters was first defined by Neyman and Scott (1948). Lancaster (2000) and Moon et al. (2015) offer rigurous treatments of the incidental parameters problem.

36 Several authors have shown the connection between the non-parametric bootstrap, the parametric (Bayesian) bootstrap and MCMC, respectively (see e.g. Rubin, 1981; Efron, 1982; Newton and Raftery, 1994; Hastie et al., 2009, Ch, 8; and Efron, 2011).

37 The results from our simulations are available from the authors upon request. These simulations did not include the null of endogeneity between explained and explanatory variables and thus do not introduce an IV structure in the model. A more detailed simulation-based analysis of the Bayesian estimator in comparison with alternative dynamic panel estimators can be found in Fernández-Amador and Oberdabernig (2018). 


$$
\begin{aligned}
\left(\epsilon_{1, i t}, \epsilon_{2, i t}\right) & \sim N(0, \Sigma) \\
\epsilon_{1 \mid 2, i t} & \sim N\left(a_{12} \epsilon_{2, i t}, \Sigma_{1 \mid 2}\right) \\
\epsilon_{2, i t} & \sim N\left(0, \Sigma_{22}\right)
\end{aligned}
$$

where again $a_{12}=\Sigma_{12} / \Sigma_{22}$ and the equations (16) and (17), for the priors of the individualspecific weights in the variance parameter, as well as the equations (10) and (11) for the prior of the degrees of freedom $\nu$ are eliminated. Therefore, the model collapses to the Bayesian IV-model proposed by Lopes and Polson (2014). The Gibbs sampling algorithm for estimating this model's posterior is simplified by deleting the steps corresponding to the parameters $\left\{\omega_{2, i}^{-1}, \omega_{1 \mid 2, i}^{-1}\right\}_{i=1, \ldots, N}$ and $\nu$.

\section{Results}

We implement two types of IV models with errors distributed as a scale mixture of normals that differ in the inclusion or exclusion of individual-specific dummy variables (DV). The $D V$-conditional heteroscedastic model includes a set of economic, political and structural controls, and individual-specific dummy variables. It constitutes a test for (fully) conditional convergence. The conditional heteroscedastic model does not include individualspecific effects and is only conditioned on economic, political and structural variables. This model provides evidence on a stronger assumption about convergence than the $D V$ conditional heteroscedastic model, as it reflects the concept of global convergence.

Table 1 summarizes the results of the $D V$-conditional heteroscedastic model. The results of the conditional heteroscedastic model (without individual-dummies) are available in Table 2. ${ }^{38}$ The four columns of the tables report the posterior means of the parameter estimates from the outcome (upper panel) and the instrumental equations (middle panel) together with the $R^{2}$, the PIPs of the regressors associated with specific-group convergence, the half-life derived from the convergence estimates (lower panel), the posterior the hyperparameter governing the weights $\nu$ associated with the country-specific variances, the Deviance Information Criterion (DIC), and the number of observations of the regressions for the four $\mathrm{CO}_{2}$ inventories $\left(\mathrm{CO}_{2}\right.$ per capita and per value added for production and consumption inventories). The asterisks next to the parameter estimates indicate whether the parameter is different from zero at the $99 \%, 95 \%$ or $90 \%$ (equal-tailed) credible interval (CI).

38 Furthermore, we report the results of the $D V$-conditional and conditional homoscedastic models (normaldistributed errors with and without individual-dummies) in Tables A.4 and A.5 in the Appendix. 
The estimated $\nu$ turn out to be very low (between 4 and 5), pointing to the existence of heteroscedasticity for each of the four inventories, for the specifications with and without individual fixed effects (tables 1 and 2, respectively). The $R^{2}$ are relatively high throughout, indicating that the included regressors explain a large part of the variation in the growth rates of all four emission inventories. ${ }^{39}$ In particular, the $D V$-conditional model explains $73 \%-74 \%$ of the variation in growth of $\mathrm{CO}_{2}$ emissions per capita and per value added embodied in production activities, while it accounts for $60 \%-66 \%$ of the variation in the growth rate of emissions per capita and per value added embodied in consumption. The explanatory power of the conditional model decreases to $26 \%-28 \%$ for production inventories, and respectively $31 \%$ and $47 \%$ for $\mathrm{CO}_{2}$ consumption intensities and $\mathrm{CO}_{2}$ consumption per capita. The difference between the $R^{2}$ of the specifications underlines the importance of country-specific steady states. However, the inspection of the DIC across specifications lends support to both $D V$-conditional and conditional models as a representation of the dynamics of carbon emissions per capita and per value added.

In all specifications we instrument the growth rate of income per capita in order to account for potential reverse causality (see Barro and Sala-i Martin, 1992). The coefficient of lagged income per capita growth, which we use as an instrument, is positive with a CI of $99 \%$ in each specification, indicating a high relevance of this variable. At the same time it is exogenous, as emission growth cannot affect lagged growth rates of income per capita. The estimate for $a_{i v}$, the strength of the correlation between the errors of the instrumental and the outcome equations is insignificant at the $90 \% \mathrm{CI}$ for all emission inventories but for carbon emissions per capita from consumption in the $D V$-conditional model.

\subsection{DV-conditional convergence}

For the $D V$-conditional convergence model in Table 1, the posterior mean of the parameter connected to lagged emissions (the convergence parameter, $\beta$ ) reveals a negative effect of lagged emissions on the average growth rate of all four emission inventories, at a CI of $99 \%$. This provides strong evidence for convergence in all four $\mathrm{CO}_{2}$ emission inventories. The magnitudes of the posterior mean of the convergence parameter are larger in absolute value for production inventories than for consumption inventories.

Given the size of the convergence parameters, it is possible to calculate the time needed for countries to halve their emissions gap towards their country-specific steady states. Assum-

39 It should be noted that Bayesian estimation does not aim at minimizing the sum of square residuals and thus, it does not maximize the $R^{2}$. However, we consider it together with the DIC when assessing how well our models fit the data and whether they can be regarded as consistent with our data. The DIC penalizes the number of parameters and is often regarded as a better measure of fit in the Bayesian context than the $R^{2}$. 
(1) $\quad(2)$

(3)

$(4)$

$\mathrm{CO}_{2}$ pc prod. $\mathrm{CO}_{2}$ pc cons. $\mathrm{CO}_{2}$ va prod. $\mathrm{CO}_{2}$ va cons.

\begin{tabular}{|c|c|c|c|c|}
\hline \multirow[b]{2}{*}{ Constant } & \multicolumn{4}{|c|}{ Outcome equation } \\
\hline & $-0.4089 * * *$ & $-0.4012 * * *$ & 0.2011 & 0.1240 \\
\hline Ln(emissions) & $-0.2009 * * *$ & $-0.1741 * * *$ & $-0.2269 * * *$ & $-0.1372 * * *$ \\
\hline $\operatorname{Ln}($ emissions $) \cdot \mathrm{EU}$ & -0.0001 & -0.0001 & -0.0005 & -0.0002 \\
\hline Ln(emissions) OECD & -0.0004 & -0.0004 & 0.0066 & 0.0004 \\
\hline $\operatorname{Ln}($ emissions $) \cdot$ Annex I & 0.0000 & 0.0000 & -0.0008 & -0.0124 \\
\hline Ln(Income pc) & $0.0932 * * *$ & $0.0769 * * *$ & -0.0187 & -0.0020 \\
\hline Income pc growth & $0.8159 * * *$ & $1.5034 * * *$ & -0.4024 & -0.1206 \\
\hline Ln(pop. density) & $-0.0750 * * *$ & -0.0423 & -0.0189 & -0.0261 \\
\hline Fossil rents & $0.0029 * *$ & 0.0019 & $0.0025 *$ & 0.0015 \\
\hline Nuclear $\%$ & $0.0007 *$ & $0.0008 *$ & 0.0005 & 0.0001 \\
\hline Fossil fuels $\%$ & $0.0007 * *$ & $0.0007 * *$ & $0.0007 *$ & 0.0002 \\
\hline Openness & -0.0001 & 0.0000 & 0.0000 & 0.0000 \\
\hline Political Regime & $-0.0017 * *$ & -0.0009 & $-0.0020 * *$ & $-0.0015 *$ \\
\hline VA energy \% & 0.0003 & 0.0004 & -0.0004 & -0.0005 \\
\hline VA light manufacturing \% & 0.0008 & 0.0002 & 0.0006 & 0.0007 \\
\hline VA heavy manufacturing $\%$ & -0.0004 & 0.0002 & $-0.0018 *$ & $-0.0020 *$ \\
\hline VA textiles $\%$ & 0.0026 & -0.0005 & $0.0070 * * *$ & 0.0016 \\
\hline VA water services $\%$ & $0.0146 *$ & 0.0083 & 0.0075 & 0.0038 \\
\hline VA construction $\%$ & $-0.0020 *$ & 0.0007 & $-0.0039 * * *$ & -0.0002 \\
\hline VA trade and transport \% & 0.0004 & 0.0001 & 0.0001 & -0.0004 \\
\hline VA other services \% & 0.0006 & -0.0007 & 0.0002 & 0.0004 \\
\hline 2004 & $0.0244 * * *$ & $0.0291 * * *$ & -0.0058 & $-0.0135 * *$ \\
\hline 2007 & 0.0064 & $0.0171 * * *$ & $-0.0244 * * *$ & $-0.0338 * * *$ \\
\hline 2011 & $0.0119 *$ & $0.0199 * * *$ & $-0.0248 * * *$ & $-0.0383 * * *$ \\
\hline 2014 & -0.0002 & 0.0102 & $-0.0485 * * *$ & $-0.0554 * * *$ \\
\hline Individual-dummies & yes & yes & yes & yes \\
\hline \multirow[t]{2}{*}{$\mathrm{R}^{2}$} & 0.7272 & 0.6513 & 0.7398 & 0.6055 \\
\hline & \multicolumn{4}{|c|}{ Instrumental equation for income pc growth } \\
\hline Constant & $0.0147 * * *$ & $0.0147 * * *$ & $0.0147 * * *$ & $0.0147 * * *$ \\
\hline Income pc growth, lagged & $0.3597 * * *$ & $0.3618 * * *$ & $0.3596 * * *$ & $0.3597 * * *$ \\
\hline$a_{i v}$ & -0.1678 & $-0.5926 * *$ & 0.1366 & -0.1570 \\
\hline $\mathrm{R}^{2}$ & 0.5328 & 0.5334 & 0.5328 & 0.5322 \\
\hline PIP EU & 0.0100 & 0.0225 & 0.0192 & 0.0223 \\
\hline PIP OECD & 0.0062 & 0.0185 & 0.1885 & 0.0192 \\
\hline PIP Annex I & 0.0229 & 0.0032 & 0.0309 & 0.5115 \\
\hline Half-life & 3.0907 & 3.6237 & 2.6934 & 4.6970 \\
\hline$\nu$ & 4.6581 & 4.8148 & 4.6797 & 4.4001 \\
\hline DIC & -3283.5 & -3212.1 & -3165.0 & -3141.1 \\
\hline $\mathrm{N}$ & 390 & 390 & 390 & 390 \\
\hline
\end{tabular}

Note: * CI 90\%, ** CI 95\%, *** CI 99\%. All variables but group dummies and income pc growth enter in lagged values. The half-life is calculated as $-\ln (0.5) /-\ln (1+\beta)$ (see Allington and McCombie, 2007). The Bayesian $R^{2}$ is the mean of the $R^{2}$ computed for each draw $q$ of the Markov chain (MC), $R_{q}^{2}$ where $R_{q}^{2}=\sum_{i, t} \hat{y}_{i t} /\left(\sum_{i, t} \hat{y}_{i t}+\sum_{i, t} \epsilon_{i t}\right)$, where $\hat{y}_{i t}$ is the estimate of $y_{i t}$ implied by the model and $\epsilon_{i t}=y_{i t}-\hat{y_{i t}}$ (Gelman et al., 2017, see). The Deviance Information Criterion (DIC) is computed as $D I C=\hat{D}_{q}+\operatorname{Var}\left(D_{q}\right) / 2$, where $D_{q}$ is the deviance measure associated with draw $q$ in the MC (see Spiegelhalter, 2002; Gelman et al., 2004, Ch. 7).

Table 1: Results scale-mixture of normals, $D V$-conditional heteroscedastic model 
ing that the average emission trajectories observed in the sample remain unchanged, the half-life of emissions amounts to 3.1 (3.6) years for $\mathrm{CO}_{2}$ per capita production (consumption) and 2.7 (4.7) years for $\mathrm{CO}_{2}$ production (consumption) per unit of value added. ${ }^{40}$ These rather fast convergence rates implied by our estimates are in line with the findings of Westerlund and Basher (2008) and Jobert et al. (2010) for $\mathrm{CO}_{2}$ per capita from production activities. Westerlund and Basher (2008) reported a half-life of $\mathrm{CO}_{2}$ emissions per capita of between 3.1 and 6.1 years in a sample of developed and developing countries. ${ }^{41}$ Jobert et al. (2010) found the half-life of $\mathrm{CO}_{2}$ emissions to be between 2.2 and 3.4 years for various OECD countries. ${ }^{42}$ Thus, our results confirm that the findings of earlier studies covering a smaller number of countries also hold for a sample of countries comprising the whole world.

There is no strong evidence for the existence of specific convergence dynamics for EU, OECD or Annex I members. The PIPs of the group-specific regressors are usually smaller than $10 \%$, with the exception of the group of OECD countries in the model for $\mathrm{CO}_{2}$ production per value added and the group of Annex I countries in the model for $\mathrm{CO}_{2}$ consumption per value added, with PIPs of $19 \%$ and 51\%, respectively. A low PIP implies that the estimation algorithm tends to exclude group-specific dynamics. Consequently, the slope estimates of the group-specific regressors are very low in magnitude and not different from zero at any of the CIs considered. Also the group-specific convergence terms with a higher PIP fail to be different from zero at any of the specified CI.

Some of the control variables capturing economic and institutional characteristics have significant effects on emission growth. Higher per capita income and a higher growth rate of per capita income are associated with higher growth rates of $\mathrm{CO}_{2}$ per capita, while $\mathrm{CO}_{2}$ intensities are not significantly affected by these variables. This highlights the role of energy - and thus energy-derived $\mathrm{CO}_{2}$ emissions - as a necessary input for production and consumption patterns. Population density has a negative effect on the growth rate of $\mathrm{CO}_{2}$ per capita. The opposite is true for the share of rents from fossil fuel production in GDP, which has a positive effect on the growth rate of both $\mathrm{CO}_{2}$ production inventories. With respect to the variables related to the composition of electricity production in an economy, a higher share of fossil fuels or nuclear sources in total electricity production is connected to a larger growth rate of $\mathrm{CO}_{2}$ emissions per capita, and in the case of fossil fuels also to a higher growth rate of $\mathrm{CO}_{2}$ production intensity. Noteworthy, trade openness does not

\footnotetext{
40 The half-life provides an indication of the speed of convergence. It is defined as the time required to eliminate half of the initial gap between actual emissions levels and the steady state. The half-life is calculated as $\frac{-\ln (0.5)}{-\ln (1+\beta)}$ (see Allington and McCombie, 2007, p. 206).

41 The half-life in their sample of developed countries was estimated to lie between 4.2 and 6.2 years; this is longer than the half-life estimated in their pooled sample including developing countries.

42 These figures correspond to estimates of conditional convergence. For unconditional convergence the authors reported a half-life between 4 and 8.5 years.
} 
affect emissions growth for any of the inventories considered. More democratic regimes are connected to lower growth rates of $\mathrm{CO}_{2}$ for all inventories but $\mathrm{CO}_{2}$ consumption per capita, suggesting that democracy may be a channel through which citizens' preferences are revealed (see Farzin and Bond, 2006). ${ }^{43}$

Regarding the sectoral shares in value added, only four sectors are relevant at a CI of at least $90 \%$. These are heavy manufacturing, which tends to reduce the growth rate of $\mathrm{CO}_{2}$ intensity, textiles, which increases the growth of $\mathrm{CO}_{2}$ production intensity, water services, which are connected to higher emission growth rates for $\mathrm{CO}_{2}$ production, and the construction sector, which lowers the growth rate of both production-based emission inventories. ${ }^{44}$ The time-dummies are different from zero at the selected CI in most cases. For carbon emissions per capita, they point towards a significant increase in emissions growth in 2004 worldwide, followed by a slight decrease afterward. For $\mathrm{CO}_{2}$ per value added, by contrast, the results indicate a global decrease in emission intensities over time.

\subsection{Conditional convergence without individual-dummies}

The $D V$-conditional model analyzed above includes individual-specific effects and is thus concerned with convergence towards individual-specific steady-states. A stronger assumption is that convergence occurs towards a common steady-state that is determined by economic and political factors. In order to test for international convergence towards a common level of emissions per capita or per value added, we also estimate models without individual-specific effects. The results from conditional models without individualdummies, displayed in Table 2, show a slightly different pattern of convergence. The convergence parameters (of lagged emissions) are still relevant at the 99\% CI for all inventories, but are substantially smaller in absolute values than in the $D V$-conditional models; they indicate a half-life of 26 (15) years for $\mathrm{CO}_{2}$ per capita production (consumption) and of 45 (44) years for $\mathrm{CO}_{2}$ production (consumption) intensities. Group-specific convergence patterns remain unimportant, with PIPs that are even lower than for the $D V$-conditional models (in most cases below 1\%).

With regard to the control variables, some turn irrelevant for explaining emissions growth - fossil rents, the share of nuclear sources in electricity production, and value added

43 The negative effect of democracy on emissions growth is not robust to using alternative measures of democracy, such as the democracy measure sourced from the FSD1289 Measures of Democracy 1810-2014 database (see Finnish Social Science Data Archive, 2018) or the average of the Freedom House indexes of political rights and civil liberties (see Freedom House, 2018). The main results are not sensitive to these alternative specifications and are also robust to the exclusion of the democracy variable.

44 The negative impact of the construction sector may be related to the low carbon intensity of this sector during the period analyzed. We take the value added share of agriculture as the benchmark sector and exclude it from the specifications in order to avoid multicollinearity. 

(1)
$(2)$
(3)
$(4)$

$\mathrm{CO}_{2}$ pc prod. $\mathrm{CO}_{2}$ pc cons. $\mathrm{CO}_{2}$ va prod. $\mathrm{CO}_{2}$ va cons.

\begin{tabular}{|c|c|c|c|c|}
\hline \multirow[b]{2}{*}{ Constant } & \multicolumn{4}{|c|}{ Outcome equation } \\
\hline & $-0.3611 * * *$ & $-0.4721 * * *$ & $-0.0938 * *$ & -0.0112 \\
\hline Ln(emissions) & $-0.0266 * * *$ & $-0.0460 * * *$ & $-0.0154 * * *$ & $-0.0158 * * *$ \\
\hline $\operatorname{Ln}($ emissions $) \cdot E U$ & 0.0001 & 0.0000 & 0.0003 & 0.0002 \\
\hline Ln(emissions) $\cdot$ OECD & 0.0002 & 0.0002 & -0.0002 & -0.0002 \\
\hline Ln(emissions $) \cdot$ Annex I & -0.0002 & -0.0003 & 0.0006 & 0.0002 \\
\hline Ln(income pc) & $0.0336 * * *$ & $0.0525 * * *$ & $0.0105 * *$ & 0.0011 \\
\hline Income pc growth & $0.7263 * * *$ & $1.0017 * * *$ & -0.1722 & 0.0095 \\
\hline Ln(pop. density) & $-0.0033 *$ & $-0.0047 * * *$ & -0.0034 & $-0.0040 * *$ \\
\hline Fossil rents & -0.0006 & -0.0009 & 0.0002 & 0.0005 \\
\hline Nuclear \% & -0.0001 & 0.0001 & 0.0001 & 0.0001 \\
\hline Fossil fuels $\%$ & $0.0002 *$ & $0.0002 *$ & 0.0001 & 0.0001 \\
\hline Openness & 0.0000 & 0.0000 & -0.0001 & 0.0000 \\
\hline Political regime & $-0.0011 * *$ & -0.0008 & -0.0008 & -0.0004 \\
\hline VA energy \% & $0.0013 *$ & $0.0014 * *$ & 0.0001 & -0.0002 \\
\hline VA light manufacturing \% & 0.0004 & -0.0005 & 0.0001 & -0.0001 \\
\hline VA heavy manufacturing $\%$ & 0.0005 & 0.0003 & -0.0003 & -0.0001 \\
\hline VA textiles $\%$ & $0.0042 * * *$ & $0.0024 * *$ & $0.0052 * * *$ & $0.0027 * *$ \\
\hline VA water services $\%$ & -0.0020 & -0.0046 & 0.0062 & -0.0051 \\
\hline VA construction \% & -0.0004 & -0.0008 & -0.0015 & 0.0002 \\
\hline VA trade and transport \% & 0.0008 & 0.0006 & -0.0001 & 0.0001 \\
\hline VA other services $\%$ & 0.0008 & 0.0004 & -0.0003 & -0.0001 \\
\hline 2004 & $0.0335 * * *$ & $0.0305 * * *$ & $0.0140 *$ & 0.0037 \\
\hline 2007 & 0.0063 & 0.0035 & 0.0042 & -0.0079 \\
\hline 2011 & $0.0115 *$ & 0.0083 & 0.0117 & -0.0039 \\
\hline 2014 & -0.0017 & -0.0089 & -0.0013 & $-0.0148 * *$ \\
\hline Individual-dummies & no & no & no & no \\
\hline \multirow[t]{2}{*}{$\mathrm{R}^{2}$} & 0.2757 & 0.4659 & 0.2554 & 0.3117 \\
\hline & \multicolumn{4}{|c|}{ Instrumental equation for income pc growth } \\
\hline Constant & $0.0145 * * *$ & $0.0147 * * *$ & $0.0146 * * *$ & $0.0147 * * *$ \\
\hline Income pc growth, lagged & $0.3620 * * *$ & $0.3594^{* * *}$ & $0.3603 * * *$ & $0.3589 * * *$ \\
\hline$a_{i v}$ & -0.0714 & 0.0114 & -0.2114 & -0.3918 \\
\hline $\mathrm{R}^{2}$ & 0.5314 & 0.5324 & 0.5319 & 0.5326 \\
\hline PIP EU & 0.0029 & 0.0020 & 0.0075 & 0.0055 \\
\hline PIP OECD & 0.0038 & 0.0095 & 0.0083 & 0.0061 \\
\hline PIP Annex I & 0.0023 & 0.0056 & 0.0322 & 0.0050 \\
\hline Half-life & 25.7100 & 14.7191 & 44.6621 & 43.5226 \\
\hline$\nu$ & 3.8105 & 4.5663 & 4.2396 & 4.6691 \\
\hline DIC & -3230.3 & -3259.2 & -3018.1 & -3223.5 \\
\hline $\mathrm{N}$ & 390 & 390 & 390 & 390 \\
\hline
\end{tabular}

Note: * CI 90\%, ** CI 95\%, *** CI 99\%. All variables but group dummies and income pc growth enter in lagged values. The half-life is calculated as $-\ln (0.5) /-\ln (1+\beta)$ (see Allington and McCombie, 2007). The Bayesian $R^{2}$ is the mean of the $R^{2}$ computed for each draw $q$ of the Markov chain (MC), $R_{q}^{2}$ where $R_{q}^{2}=\sum_{i, t} \hat{y}_{i t} /\left(\sum_{i, t} \hat{y}_{i t}+\sum_{i, t} \epsilon_{i t}\right)$, where $\hat{y}_{i t}$ is the estimate of $y_{i t}$ implied by the model and $\epsilon_{i t}=y_{i t}-\hat{y_{i t}}$ (Gelman et al., 2017, see). The Deviance Information Criterion (DIC) is computed as $D I C=\hat{D}_{q}+\operatorname{Var}\left(D_{q}\right) / 2$, where $D_{q}$ is the deviance measure associated with draw $q$ in the MC (see Spiegelhalter, 2002; Gelman et al., 2004, Ch. 7).

Table 2: Results scale-mixture of normals, conditional heteroscedastic model 
shares of heavy manufacturing, water services and construction for all inventories, as well as political regime and the share of fossil fuels in electricity production for $\mathrm{CO}_{2}$ intensities. Some others gain relevance, namely population density for consumption inventories, the value added share of the energy sector for per capita emissions, and the value added share of the textile sector for all inventories. Furthermore, income per capita is now relevant for all inventories but $\mathrm{CO}_{2}$ consumption intensity. ${ }^{45}$

To sum up, our findings provide strong evidence for rather fast rates of convergence towards country-specific steady states for all four $\mathrm{CO}_{2}$ inventories ( $D V$-conditional model). International convergence towards global steady states determined by political and economic structures proceeds at a much slower pace (conditional model). Although some previous studies have found evidence for group-specific convergence patterns for OECD and EU members (e.g. Aldy, 2006; Nguyen, 2005; Ordás Criado and Grether, 2011; Panopoulou and Pantelidis, 2009; Westerlund and Basher, 2008), none of our models provides evidence for differences in convergence dynamics implied by membership in the OECD, EU, or Annex I of the Kyoto Protocol. Therefore, climate change policies of industrialized countries such as the OECD or the EU have not been effective in accelerating emission convergence among developed economies (see also Westerlund and Basher, 2008, who found slower convergence for OECD countries). Furthermore, the binding commitments of the Kyoto Protocol have been largely ineffective in accelerating emission convergence among Annex I countries (see also Ordás Criado and Grether, 2011). ${ }^{46}$

\section{Conclusion and discussion}

We tested for international convergence of $\mathrm{CO}_{2}$ per capita and per value added derived from production and consumption patterns across a global sample of countries during 1997-2014. In so doing, we put forward a Bayesian test for convergence that is robust to cross-sectional heteroscedasticity, accounts for endogeneity between the growth rate of $\mathrm{CO}_{2}$ emissions and economic growth, and allows for the existence of group-specific convergence among members of the EU, the OECD, and the Annex I of the Kyoto Protocol.

Our findings suggest that all four emission inventories converge towards country-specific steady states. The short half-lives calculated show that emissions per capita as well as

45 The results of the models without individual-dummies could be affected by omitted variables and should be taken with care (see Barro and Sala-i Martin, 2004).

46 Tables A.4 and A.5 in the Appendix report the results for the models with homoscedastic errors. The main results do not change qualitatively. The only important qualitative change is that income growth becomes significant for emissions per value added in the $D V$-conditional models. The convergence coefficient only changes slightly, though this change is amplified in the half-lives, decreasing them in the specifications for emissions per value added in the conditional models. 
emission intensities are close to their country-specific steady states. Production-based inventories show a shorter half-life (2.7-3.1 years) than consumption-based inventories (3.6-4.7 years). The finding that for production inventories $\mathrm{CO}_{2}$ intensity converges faster than $\mathrm{CO}_{2}$ per capita is in line with the results of Anjum et al. (2014). For $\mathrm{CO}_{2}$ consumption inventories the reverse is true, what provides a first indication that, internationally, $\mathrm{CO}_{2}$ intensities converge more slowly than $\mathrm{CO}_{2}$ per capita.

In fact, a much slower pace of $\mathrm{CO}_{2}$ convergence across countries - towards global steady states that are determined by economic and political structures - is detected for all four emission inventories. Emissions per capita embodied in consumption show substantially faster global conditional converge (with a half-life of 14.7 years) than emissions per capita embodied in production (25.7 years). This is consistent with converging consumption bundles across countries as a result of increasing globalization and the homogenization of consumer tastes, while the slower cross-country convergence of production-based emissions can be related to the long-run nature of structural transformations of production patterns. Emission intensities converge towards global steady states at an even slower pace, which is similar for production and consumption inventories (implying half-lives of 44-45 years). The very slow pace of global convergence of emission intensities indicates that technology transfers across countries have been rather limited and cannot explain the faster rate of convergence of emissions per capita. Thus, the faster convergence of emissions per capita is likely to be driven by some of the conditioning variables, possibly income growth. Various empirical studies have shown that income and $\mathrm{CO}_{2}$ emissions are positively related (e.g. Azomahou et al., 2006; Holtz-Eakin and Selden, 1995; Fernández-Amador et al., 2017). To the extent that the economic crisis of 2008 has led to a decline in economic growth in high-income countries, whereas countries with lower income levels were able to catch up, this catch-up process could have sped up the international convergence of per capita emissions (see also Brock and Taylor, 2010). ${ }^{47}$

Actual levels of carbon emissions have proven to be unsustainable. Our results indicate that higher income growth is related to a higher growth-rate of emissions per capita, while it is not connected to a decrease in emission intensities. The evidence for convergence does not automatically imply convergence towards steady states that are sustainable, as highlighted by Brock and Taylor (2010) and Ordás Criado et al. (2011). Moreover, the evidence for fast country-specific convergence towards not sustainable steady states underlines the current incompatibility between economic growth and the $2^{\circ} \mathrm{C}$ target, and the need for further abatement and mitigation policies to keep global warming under control while maintaining reasonable economic growth rates.

\footnotetext{
47 A more detailed analysis of this mechanism is out of the scope of this paper.
} 
The historical responsibility for atmospheric $\mathrm{CO}_{2}$ concentrations corresponds to developed economies. However, those economies, represented in our sample by three groups-OECD, EU, and the countries that ratified the Annex I of the Kyoto Protocol-have not experienced faster group convergence. This lack of specific patterns of convergence among developed economies, despite the environmental policies implemented in these countries during the period of analysis after the Kyoto Protocol, shows the difficulties in achieving effective agreements and policies to take action against global warming.

The slow pace of international convergence of emissions and the limited extent of international technology diffusion pose doubts on the feasibility of the agreed sustainability targets, unless a significant change in the international institutional framework takes place, and new, stronger abatement policies are implemented. Also, much faster transfers of green technologies will be necessary.

The lack of a stabilization of emissions in industrialized economies at sustainable emission levels may discourage developing economies to accept a cap on emissions. In addition, the evidence found for country-specific steady states in emissions points to significant transaction costs connected to the design of multilateral policy frameworks aimed at global emissions reduction. Even though there is an urgency for multilateral approaches to fight climate change that encompass developed and developing countries, developed economies should foster further national environmental policies to promote carbon efficiency and less polluting sources of energy in order to reinforce the international action against global warming. 
A Online Appendix 


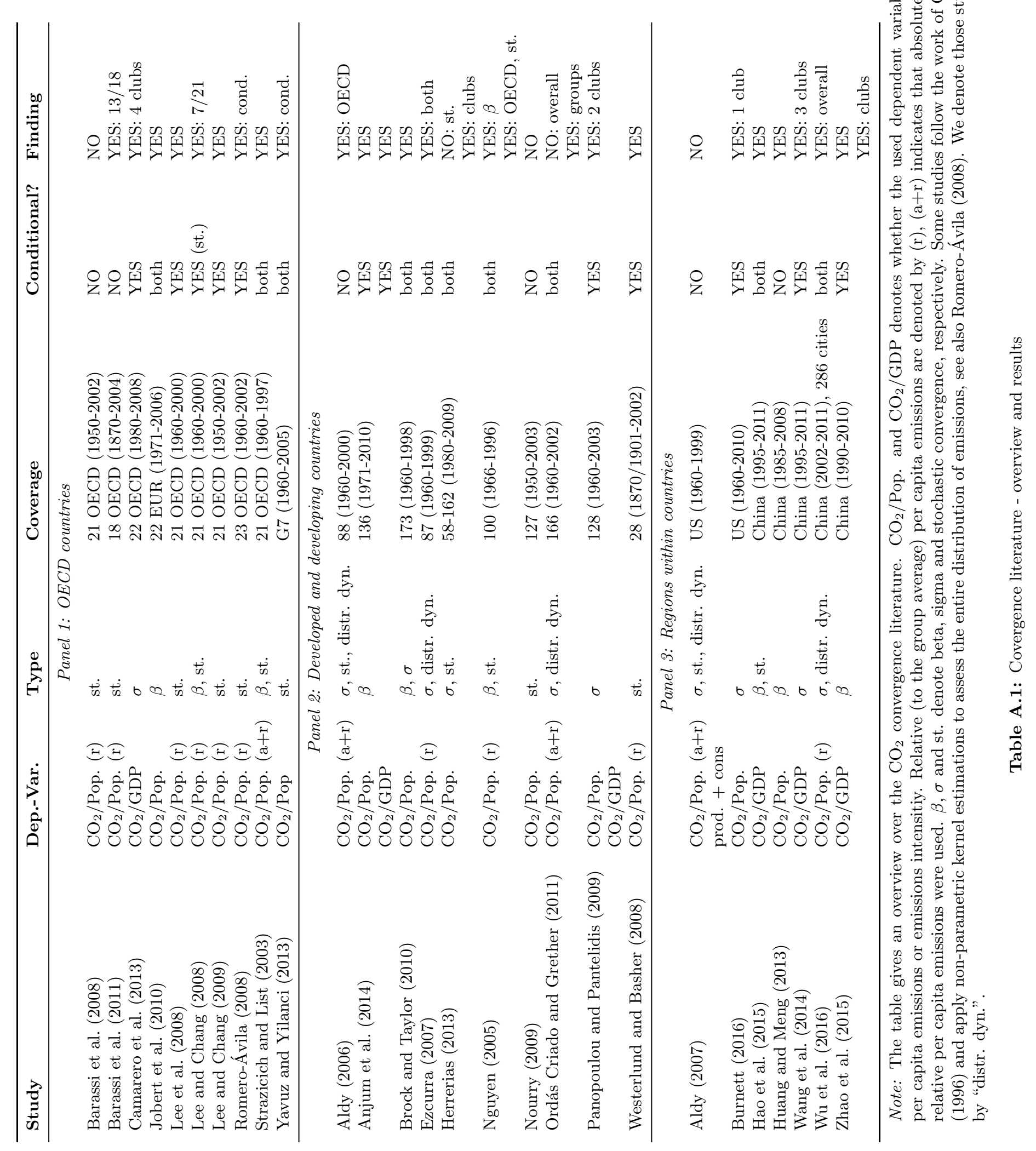




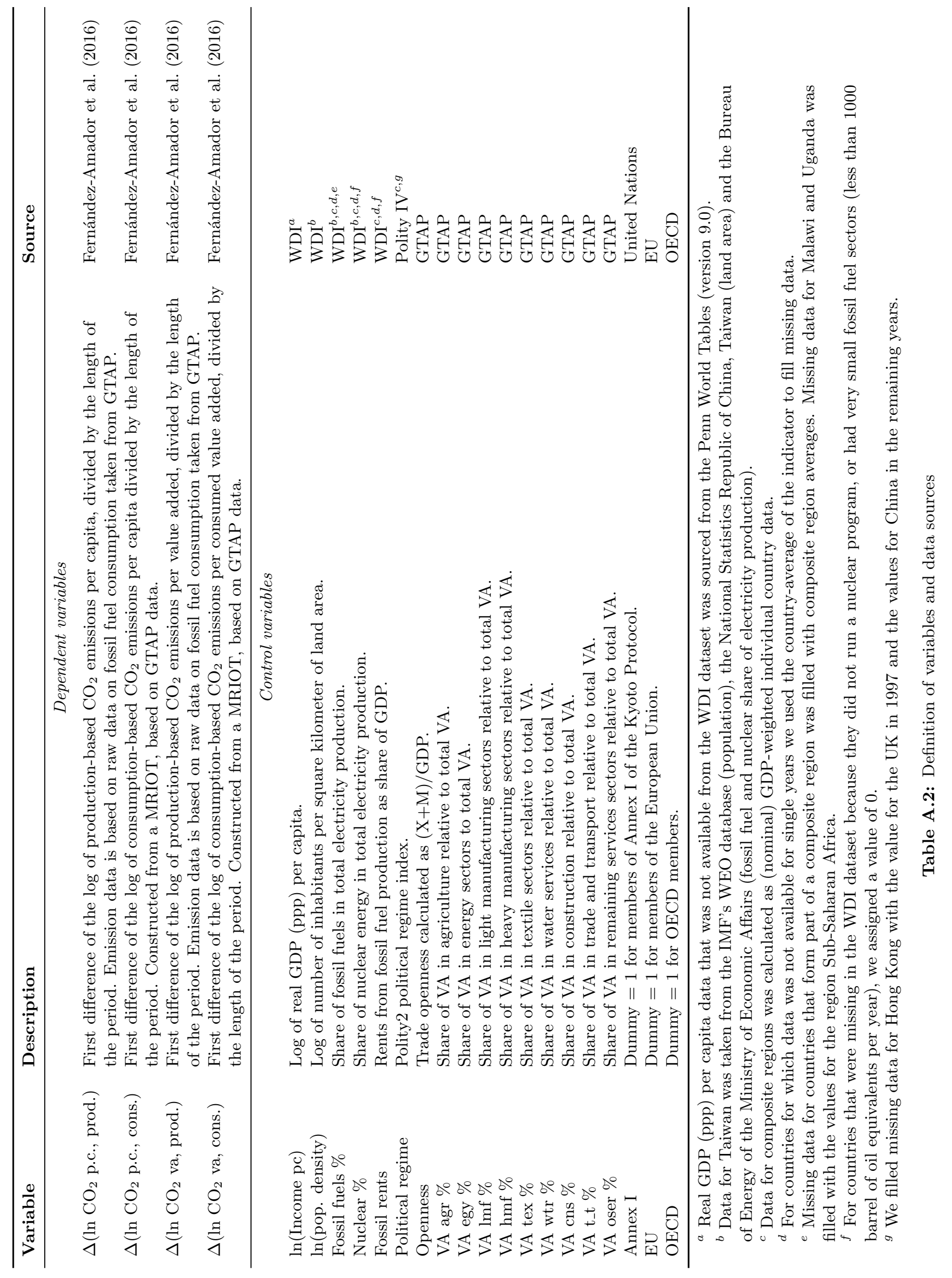




\begin{tabular}{|c|c|c|c|c|c|}
\hline & $\mathrm{N}$ & Mean & Std. dev & Min & Max \\
\hline \multicolumn{6}{|c|}{ Dependent variables } \\
\hline Growth $\mathrm{CO}_{2}$ pc prod. & 390 & 0.0066 & 0.0513 & -0.3302 & 0.2667 \\
\hline $\mathrm{O}_{2}$ pc cons. & 390 & 0.0108 & 0.0500 & -0.1884 & 0.2156 \\
\hline Growth $\mathrm{CO}_{2}$ va prod. & 390 & -0.0207 & 0.0577 & -0.3572 & 0.2930 \\
\hline Growth $\mathrm{CO}_{2}$ va cons. & 390 & -0.0167 & 0.0431 & -0.2388 & 0.2311 \\
\hline \multicolumn{6}{|c|}{ Control variables } \\
\hline $\mathrm{Ln}\left(\mathrm{CO}_{2}\right.$ pc prod. $)$ & 390 & 1.2085 & 1.4009 & -2.6795 & 3.5778 \\
\hline $\mathrm{Ln}\left(\mathrm{CO}_{2}\right.$ pc cons. $)$ & 390 & 1.3226 & 1.3019 & -2.0201 & 3.6115 \\
\hline $\mathrm{Ln}\left(\mathrm{CO}_{2}\right.$ va prod. $)$ & 390 & -0.1894 & 0.7446 & -1.9935 & 1.8894 \\
\hline $\mathrm{Ln}\left(\mathrm{CO}_{2}\right.$ va cons. $)$ & 390 & -0.0923 & 0.5449 & -1.1193 & 1.6246 \\
\hline $\mathrm{EU}$ & 390 & 0.3128 & & 0.0000 & 1.0000 \\
\hline $\mathrm{OH}$ & 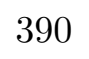 & 0.3872 & & 0.0000 & 1.0000 \\
\hline Anne & 390 & 0.3718 & & 0.0000 & 1.0000 \\
\hline $\operatorname{Ln}($ ir & 390 & 9.4981 & 1.1 & 6.2054 & 11.4913 \\
\hline Incom & 390 & 0.0260 & 0.0 & -0.1240 & 0.1194 \\
\hline lagged & 39 & 0.0278 & & -0.1240 & 0.1194 \\
\hline Ln(pop. density) & 390 & 4.3048 & 77 & 0.8798 & 8.9042 \\
\hline Openness & 390 & 0.8212 & 53 & 0.1761 & 3.2739 \\
\hline Pol & 390 & 6.2256 & 5.1222 & -7.0000 & 10.0000 \\
\hline Nucl & 390 & 0.0984 & & 0.0000 & 0.8357 \\
\hline Fos & & & & & 1.0000 \\
\hline ents $\%$ & 390 & 0.0275 & 0. & 0.0000 & 0.4056 \\
\hline VA agriculture $\%$ & 390 & 0.0908 & 0.0883 & 0.0017 & 0.5529 \\
\hline VA energy \% & 390 & 0.0688 & 0.0736 & 0.0000 & 0.4313 \\
\hline VA light manı & 39 & 0.0757 & & .0246 & 0.3135 \\
\hline VA heavy manufacturing $\%$ & 390 & & & 0.0098 & 0.4428 \\
\hline VA textiles $\%$ & 390 & 0.0208 & 0.0193 & 0.0000 & 0.1267 \\
\hline VA water services $\%$ & 390 & 0.0037 & 0.0031 & 0.0000 & 0.0272 \\
\hline VA construction $\%$ & 39 & 0.0618 & 0.0255 & 0.0023 & 0.1742 \\
\hline VA trade and transport $\%$ & 39 & 0.1901 & 0.0663 & 0.0243 & 0.5138 \\
\hline VA other services $\%$ & 390 & 0.3820 & 0.1193 & 0.1379 & 0.6299 \\
\hline
\end{tabular}

Table A.3: Descriptive statistics 
(1) $\quad(2)$

(3)

$(4)$

$\mathrm{CO}_{2}$ pc prod. $\mathrm{CO}_{2}$ pc cons. $\mathrm{CO}_{2}$ va prod. $\mathrm{CO}_{2}$ va cons.

\begin{tabular}{|c|c|c|c|c|}
\hline \multirow[b]{2}{*}{ Constant } & \multicolumn{4}{|c|}{ Outcome equation } \\
\hline & $-0.3886 * * *$ & $-0.3977 * * *$ & 0.1904 & 0.1299 \\
\hline $\operatorname{Ln}($ emissions $)$ & $-0.2080 * * *$ & $-0.1792 * * *$ & $-0.2161 * * *$ & -0.1276 *** \\
\hline $\operatorname{Ln}($ emissions $) \cdot \mathrm{EU}$ & -0.0004 & -0.0010 & -0.0005 & -0.0004 \\
\hline Ln(emissions) $\cdot$ OECD & 0.0000 & -0.0002 & 0.0034 & 0.0003 \\
\hline Ln(emissions)·Annex I & -0.0003 & 0.0007 & 0.0001 & -0.0013 \\
\hline Ln(income pc) & $0.0982 * * *$ & $0.0790 * * *$ & -0.0215 & -0.0004 \\
\hline Income pc growth & $0.6360 * *$ & $1.1148 * * *$ & $-0.6090 * *$ & $-0.5573 * *$ \\
\hline Ln(pop. density) & $-0.0877 * * *$ & -0.0406 & -0.0107 & -0.0148 \\
\hline Fossil rents & $0.0031 * *$ & 0.0015 & $0.0028 *$ & 0.0005 \\
\hline Nuclear \% & $0.0009 * *$ & 0.0008 & 0.0003 & 0.0000 \\
\hline Fossil fuels $\%$ & $0.0008 * *$ & $0.0008 *$ & 0.0004 & 0.0001 \\
\hline Openness & 0.0000 & 0.0000 & -0.0001 & -0.0001 \\
\hline Political regime & $-0.0017 * *$ & 0.0002 & $-0.0018 *$ & -0.0013 \\
\hline VA energy \% & -0.0003 & 0.0006 & -0.0002 & -0.0003 \\
\hline VA light manufacturing $\%$ & 0.0007 & -0.0012 & 0.0007 & -0.0007 \\
\hline VA heavy manufacturing $\%$ & -0.0007 & 0.0005 & -0.0015 & $-0.0026 * * *$ \\
\hline VA textiles \% & 0.0032 & -0.0009 & $0.0094 * * *$ & 0.0037 \\
\hline VA water services $\%$ & $0.0185 * *$ & 0.0131 & 0.0120 & 0.0071 \\
\hline VA construction \% & $-0.0028 * * *$ & 0.0006 & $-0.0045 * * *$ & -0.0002 \\
\hline VA trade and transport $\%$ & 0.0004 & -0.0002 & 0.0007 & -0.0004 \\
\hline VA other services \% & 0.0002 & -0.0010 & 0.0004 & -0.0006 \\
\hline 2004 & $0.0311 * * *$ & $0.0286 * * *$ & -0.0025 & $-0.0104 *$ \\
\hline 2007 & $0.0146 * *$ & $0.0191 * * *$ & $-0.0186 * *$ & $-0.0246 * * *$ \\
\hline 2011 & $0.0213 * * *$ & $0.0262 * * *$ & $-0.0177 * *$ & -0.0246 *** \\
\hline 2014 & 0.0077 & 0.0149 & $-0.0436 * * *$ & $-0.0448^{* * *}$ \\
\hline Individual-dummies & yes & yes & yes & yes \\
\hline \multirow[t]{2}{*}{$\mathrm{R}^{2}$} & 0.7382 & 0.6513 & 0.7343 & 0.5941 \\
\hline & \multicolumn{4}{|c|}{ Instrumental equation for income pc growth } \\
\hline Constant & $0.0163 * * *$ & $0.0163 * * *$ & $0.0163 * * *$ & $0.0163 * * *$ \\
\hline Income pc growth, lagged & $0.3502 * * *$ & $0.3513 * * *$ & $0.3506 * * *$ & $0.3504 * * *$ \\
\hline$a_{i v}$ & -0.0285 & -0.2378 & 0.2592 & 0.2097 \\
\hline $\mathrm{R}^{2}$ & 0.5546 & 0.5547 & 0.5546 & 0.5546 \\
\hline PIP EU & 0.0069 & 0.0086 & 0.0190 & 0.0168 \\
\hline PIP OECD & 0.0087 & 0.0257 & 0.1069 & 0.0368 \\
\hline PIP Annex I & 0.0064 & 0.0040 & 0.0155 & 0.0546 \\
\hline Half-life & 2.9724 & 3.5100 & 2.8469 & 5.0777 \\
\hline DIC & -3298.3 & -3172.3 & -3164.3 & -3187.2 \\
\hline $\mathrm{N}$ & 390 & 390 & 390 & 390 \\
\hline
\end{tabular}

Note: * CI 90\%, ** CI 95\%, *** CI 99\%. All variables but group dummies and income pc growth enter in lagged values. The half-life is calculated as $-\ln (0.5) /-\ln (1+\beta)$ (see Allington and McCombie, 2007). The Bayesian $R^{2}$ is the mean of the $R^{2}$ computed for each draw $q$ of the Markov chain (MC), $R_{q}^{2}$ where $R_{q}^{2}=\sum_{i, t} \hat{y}_{i t} /\left(\sum_{i, t} \hat{y}_{i t}+\sum_{i, t} \epsilon_{i t}\right)$, where $\hat{y}_{i t}$ is the estimate of $y_{i t}$ implied by the model and $\epsilon_{i t}=y_{i t}-\hat{y_{i t}}$ (Gelman et al., 2017, see). The Deviance Information Criterion (DIC) is computed as $D I C=\hat{D}_{q}+\operatorname{Var}\left(D_{q}\right) / 2$, where $D_{q}$ is the deviance measure associated with draw $q$ in the MC (see Spiegelhalter, 2002; Gelman et al., 2004, Ch. 7).

Table A.4: Results normal distribution, DV-conditional homoscedastic model 
(1) $\quad(2)$

(3)

$(4)$

$\mathrm{CO}_{2}$ pc prod. $\mathrm{CO}_{2}$ pc cons. $\mathrm{CO}_{2}$ va prod. $\mathrm{CO}_{2}$ va cons.

\begin{tabular}{|c|c|c|c|c|}
\hline \multirow[b]{2}{*}{ Constant } & \multicolumn{4}{|c|}{ Outcome equation } \\
\hline & $-0.4482 * * *$ & $-0.4902 * * *$ & $-0.0866 * *$ & -0.0182 \\
\hline Ln(emissions) & $-0.0345 * * *$ & $-0.0478 * * *$ & $-0.0221 * * *$ & $-0.0184 * * *$ \\
\hline $\operatorname{Ln}($ emissions $) \cdot E U$ & 0.0001 & 0.0001 & 0.0002 & 0.0002 \\
\hline $\operatorname{Ln}($ emissions $) \cdot$ OECD & 0.0006 & 0.0005 & 0.0001 & 0.0003 \\
\hline $\operatorname{Ln}($ emissions $) \cdot A n n e x ~ I$ & 0.0005 & 0.0001 & -0.0001 & 0.0002 \\
\hline Ln(income pc) & $0.0467 * * *$ & $0.0544 * * *$ & $0.0123^{* *}$ & 0.0036 \\
\hline Income pc growth & $0.9594 * * *$ & $0.9677 * * *$ & 0.0951 & 0.0197 \\
\hline Ln(pop. density) & $-0.0057 * * *$ & $-0.0045 * *$ & $-0.0080 * * *$ & $-0.0059 * * *$ \\
\hline Fossil rents & 0.0002 & -0.0008 & 0.0009 & 0.0004 \\
\hline Nuclear $\%$ & -0.0001 & 0.0000 & 0.0001 & 0.0001 \\
\hline Fossil fuels $\%$ & $0.0003 * *$ & $0.0002 *$ & $0.0002 *$ & $0.0002 *$ \\
\hline Openness & -0.0001 & 0.0000 & $-0.0001 *$ & -0.0001 \\
\hline Political regime & $-0.0015 * *$ & -0.0005 & $-0.0016 * *$ & $-0.0010 *$ \\
\hline VA energy $\%$ & 0.0002 & $0.0013^{*}$ & -0.0011 & -0.0004 \\
\hline VA light manufacturing $\%$ & -0.0002 & -0.0006 & -0.0004 & -0.0002 \\
\hline VA heavy manufacturing $\%$ & 0.0006 & 0.0003 & -0.0003 & -0.0004 \\
\hline VA textiles $\%$ & $0.0051 * * *$ & $0.0029 * *$ & $0.0059 * * *$ & $0.0028 *$ \\
\hline VA water services $\%$ & -0.0015 & -0.0033 & 0.0035 & -0.0056 \\
\hline VA construction $\%$ & -0.0011 & -0.0005 & $-0.0022 *$ & 0.0004 \\
\hline VA trade and transport $\%$ & 0.0005 & 0.0003 & 0.0000 & 0.0000 \\
\hline VA other services $\%$ & 0.0006 & 0.0004 & -0.0005 & -0.0002 \\
\hline 2004 & $0.0476 * * *$ & $0.0339 * * *$ & $0.0239 * * *$ & 0.0051 \\
\hline 2007 & 0.0111 & 0.0085 & 0.0120 & -0.0051 \\
\hline 2011 & $0.0178 * *$ & $0.0145^{* *}$ & $0.0185 *$ & -0.0025 \\
\hline 2014 & 0.0028 & -0.0053 & 0.0055 & $-0.0133 *$ \\
\hline Individual-dummies & no & no & no & no \\
\hline \multirow[t]{2}{*}{$\mathrm{R}^{2}$} & 0.3669 & 0.4745 & 0.3170 & 0.3311 \\
\hline & \multicolumn{4}{|c|}{ Instrumental equation for income pc growth } \\
\hline Constant & $0.0163 * * *$ & $0.0163 * * *$ & $0.0163 * * *$ & $0.0163 * * *$ \\
\hline Income pc growth, lagged & $0.3504 * * *$ & $0.3500 * * *$ & $0.3506 * * *$ & $0.3503 * * *$ \\
\hline$a_{i v}$ & -0.2363 & $0.0405 * * *$ & -0.5033 & -0.4178 \\
\hline $\mathrm{R}^{2}$ & 0.5546 & 0.5545 & 0.5546 & 0.5546 \\
\hline PIP EU & 0.0084 & 0.0048 & 0.0104 & 0.0064 \\
\hline PIP OECD & 0.0032 & 0.0064 & 0.0097 & 0.0074 \\
\hline PIP Annex I & 0.0034 & 0.0053 & 0.0144 & 0.0063 \\
\hline Half-life & 19.7426 & 14.1516 & 31.0163 & 37.3234 \\
\hline DIC & -3096.9 & -3184.0 & -2933.1 & -3161.6 \\
\hline $\mathrm{N}$ & 390 & 390 & 390 & 390 \\
\hline
\end{tabular}

Note: * CI 90\%, ** CI 95\%, *** CI 99\%. All variables but group dummies and income pc growth enter in lagged values. The half-life is calculated as $-\ln (0.5) /-\ln (1+\beta)$ (see Allington and McCombie, 2007). The Bayesian $R^{2}$ is the mean of the $R^{2}$ computed for each draw $q$ of the Markov chain (MC), $R_{q}^{2}$ where $R_{q}^{2}=\sum_{i, t} \hat{y}_{i t} /\left(\sum_{i, t} \hat{y}_{i t}+\sum_{i, t} \epsilon_{i t}\right)$, where $\hat{y}_{i t}$ is the estimate of $y_{i t}$ implied by the model and $\epsilon_{i t}=y_{i t}-\hat{y_{i t}}$ (Gelman et al., 2017, see). The Deviance Information Criterion (DIC) is computed as $D I C=\hat{D}_{q}+\operatorname{Var}\left(D_{q}\right) / 2$, where $D_{q}$ is the deviance measure associated with draw $q$ in the MC (see Spiegelhalter, 2002; Gelman et al., 2004, Ch. 7).

Table A.5: Results normal distribution, conditional homoscedastic model 


\section{References}

Aichele, R., Felbermayr, G., 2015. Kyoto and carbon leakage: An empirical analysis of the carbon content of bilateral trade. The Review of Economics and Statistics 97(1), 104-115.

Aldy, J., 2006. Per capita carbon dioxide emissions: Convergence or divergence? Environmental \& Resource Economics 33, 533-555.

Aldy, J. E., 2007. Divergence in state-level per capita carbon dioxide emissions. Land Economics 83, $353-369$.

Allington, N. F., McCombie, J. S., 2007. Economic Growth: New Directions in Theory and Policy. Edward Elgar Publishing Limited, Ch. Economic growth and beta-convergence in the East European Transition Economies, pp. 200-222.

Andrews, D., Mallows, C., 1974. Scale mixtures of normal distributions. Journal of the Royal Statistical Society. Series B 36, 99-102.

Anjum, Z., Burke, P., Gerlagh, R., Stern, D., 2014. Modeling the emissions-income relationship using long-run growth rates. CCEP Working Paper 1403.

Arellano, M., Bond, S., 1991. Some tests of specification for panel data: Monte carlo evidence and an application to employment equations. The Review of Economic Studies 58 (2), 277-297.

Aslanidis, A., Iranzo, S., 2009. Environment and development: Is there a Kuznets curve for $\mathrm{CO}_{2}$ emissions? Applied Economics 41 (6), 803-810.

Azomahou, T., Laisney, F., Van, P. N., 2006. Economic development and co2 emissions: A nonparametric panel approach. Journal of Public Economics 90 (6), 1347 - 1363.

URL http://www.sciencedirect.com/science/article/pii/S0047272705001301

Babiker, M. H., 2005. Climate change policy, market structure, and carbon leakage. Journal of International Economics 65, 421-445.

Barassi, M., Cole, M. A., Elliott, R. J., 2008. Stochastic divergence or convergence of per capita carbon dioxide emissions: Re-examining the evidence. Environmental and Resource Economics 40, 121-137.

Barassi, M. R., Cole, M. A., Elliott, R. J., 2011. The stochastic convergence of $\mathrm{CO}_{2}$ emissions: A long memory approach. Environmental and Resource Economics 49, 367-385.

Barro, J., Sala-i Martin, X., 1992. Convergence. Journal of Political Economy 100 (2), 223-251.

Barro, R., 1991. Economic growth in a cross section of countries. The Quarterly Journal of Economics 106 (2), 407-443.

Barro, R., Sala-i Martin, X., 2004. Economic Growth, second edition. The MIT Press, Massachusetts.

Baumol, W., 1986. Productivity growth, convergence, and welfare: What the long-run data show. The American Economic Review 76 (5), 1072-1085.

Bernard, A., Durlauf, S., 1996. Interpreting tests of the convergence hypothesis. Journal of Econometrics $71(1-2), 161-173$. 
Blundell, R., Bond, S., 1998. Initial conditions and moment restrictions in dynamic panel data models. Journal of Econometrics 87 (1), 115-143.

Brock, W., Taylor, M., 2010. The Green Solow Model. Journal of Economic Growth 15, 127 - 153.

Bruno, G. S., 2005. Approximating the bias of the lsdv estimator for dynamic unbalanced panel data models. Economics Letters 87 (3), 361-366.

Burnett, J., 2016. Club convergence and clustering of U.S. energy-related $\mathrm{CO}_{2}$ emissions. Resource and Energy Economics 46, 62-84.

Camarero, M., Castillo, J., Picazo, A. J., Tamarit, C., 2013. Eco-efficiency and convergence in OECD countries. Environmental and Resource Economics 55, 87-106.

Carlino, G., Mills, L., 1993. Are U.S. regional incomes converging? A time series analysis. Journal of Monetary Economics 32 (2), 335-346.

Dasgupta, S., Laplante, B., Wang, H., Wheeler, D., 2002. Confronting the environmental Kuznets curve. The Journal of Economic Perspectives 16, 147-168.

De Vos, I., Everaert, G., Ruyssen, I., 2015. Bootstrap-based bias correction and inference for dynamic panels with fixed effects. The Stata Journal 15 (4), 986-1018.

Ding, P., 2016. On the conditional distribution of the multivariate t distribution. The American Statistician $70,293-295$.

Efron, B., 1982. The Jacknife, the bootstrap and other resampling plans. Vol. 38 of CBMS-NSF Regional Conference Series in Applied Mathematics. Society for Industrial and Applied Mathematics (SIAM), Philadelphia.

Efron, B., 2011. The bootstrap and markov chain monte carlo. Journal of Biopharm Statistics 21, $152-162$.

Evans, P., Karras, G., 1996. Convergence revisited. Journal of Monetary Economics 37 (2), 249-265.

Everaert, G., Pozzi, L., 2007. Bootstrap-based bias correction for dynamic panel. Journal of Economic Dynamics and Control 31, 1160-1184.

Ezcurra, R., 2007. Is there cross-country convergence in carbon dioxide emissions? Energy Policy 35, $1363-1372$.

Farzin, Y., Bond, C., 2006. Democracy and environmental quality. Journal of Development Economics 81, $213-235$.

Fernández-Amador, O., Francois, J. F., Oberdabernig, D. A., Tomberger, P., 2017. Carbon dioxide emissions and economic growth: An assessment based on production and consumption emission inventories. Ecological Economics 135, 269-279.

Fernández-Amador, O., Francois, J. F., Tomberger, P., 2016. Carbon dioxide emissions and international trade at the turn of the millennium. Ecological Economics 125, 14-26.

Fernández-Amador, O., Oberdabernig, D., 2018. Bayesian estimation of dynamic panels, mimeo.

Finnish Social Science Data Archive, 2018. Measures of democracy 1810-2018. 
Freedom House, 2018. Country and territory ratings and statuses, 1973-2018.

Friedman, M., 1992. Do old fallacies ever die? Journal of Economic Literature 30 (4), 2129-2132.

Gelman, A., Carlin, J. B., Stern, H. S., Rubin, D. B., 2004. Bayesian Data Analysis: Second Edition. Texts in Statistical Science, CRC Press.

Gelman, A., Goodrich, B., Gabry, J., Ali, I., Nov. 2017. R-squared for bayesian regression models, unpublished manuscript, Department of Statistics, Columbia University.

George, E. I., McCulloch, R. E., 1993. Variable selection via gibbs sampling. Journal of the American Statistical Association 88, 881-889.

Geweke, J., 1993. Bayesian treatment of the independent Student-t linear model. Journal of Applied Econometrics 8, S19-S40.

Grossman, G., Helpman, E., 1995. Handbook of International Economics. Amsterdam: North-Holland, Ch. Chapter 25: Technology and trade.

Hao, Y., Liao, H., Wei, Y.-M., 2015. Is China's carbon reduction target allocation reasonable? An analysis based on carbon intensity convergence. Applied Energy 142, 229-239.

Hastie, T., Tibshirani, R., Friedman, J., 2009. The elements of statistical learning, 2nd Edition. Series in Statistics. Springer.

Herrerias, M., 2013. The environmental convergence hypothesis: Carbon dioxide emissions according to the source of energy. Energy Policy 61, 1140 - 1150.

Holtz-Eakin, D., Selden, T. M., 1995. Stoking the fires? $\mathrm{co}_{2}$ emissions and economic growth. Journal of Public Economics 1995, 85-101.

Hsiao, C., Pesaran, H., Tahmiscioglu, K., 1999. Analysis of Panels and Limited Dependent Variable Models. Cambridge University Press, Cambridge, Ch. Bayes estimation of short-run coefficients in dynamic panel data models, pp. 268-296.

Huang, B., Meng, L., 2013. Convergence of per capita carbon dioxide emissions in urban China: A spatiotomporal perspective. Applied Geography 40, 21-29.

Jobert, T., Karanfil, F., Tykhonenko, A., 2010. Convergence of per capita carbon dioxide emissions in the EU: Legend or reality? Energy Economics 32, 1364-1373.

Kaika, D., Zervas, E., 2010. The environmental Kuznets Curve (EKC) theory - Part A: Concept, causes and the $\mathrm{CO}_{2}$ emissions case. Energy Policy 62, 1392-1402.

Kiviet, J. F., 1995. On bias, inconsistency, and efficiency of various estimators in dynamic panel data models. Journal of Econometrics 68 (1), 53-78.

Kleibergen, F., Zivot, E., 2003. Bayesian and classical approaches to instrumental variable regression. Journal of Econometrics 114, 29-72.

Knutti, R., R. J. S. J., Fischer, E., 2015. A scientific critique of the two-degree climate change target. Nature Geoscience 9, 13-19. 
Koop, G., 2003. Bayesian Econometrics. John Wiley \& Sons.

Lancaster, T., 2000. The incidental parameter problem since 1948. Journal of Econometrics 95, 391-413.

Lancaster, T., 2008. An Introduction to Modern Bayesian Econometrics. Blackwell Publishing.

Lee, C.-C., Chang, C.-P., 2008. New evidence on the convergence of per capita carbon dioxide emissions from panel seemingly unrelated regressions augmented Dickey-Fuller tests. Energy 33, 1468-1475.

Lee, C.-C., Chang, C.-P., 2009. Stochastic convergence of per capita carbon dioxide emissions and multiple structural breaks in OECD countries. Economic Modelling 26, 1375-1381.

Lee, C.-C., Chang, C.-P., Chen, P.-F., 2008. Do $\mathrm{CO}_{2}$ emission levels converge among 21 OECD countries? New evidence from unit root structural break tests. Applied Economics Letters 15, 551-556.

Lopes, H., Polson, N., 2014. Bayesian instrumental variables: Priors and likelihoods. Econometric Reviews 33, 100-121.

Maddala, G., Hu, W., 1996. The Econometrics of Panel Data: a Handbook of Theory with Applications, 2nd Edition. Kluwer Academic Publishers, Boston, Ch. The pooling problem, pp. 307-322.

Mankiw, N., Romer, D., Weil, D., 1992. A contribution to the empirics of economic growth. The Quarterly Journal of Economics 107 (2), 407-437.

Moon, H., Perron, B., Phillips, P., 2015. Incidental Parameters and Dynamic Panel Modeling. Oxford University Press, Oxford, Ch. 4, pp. 111-148.

Newton, M., Raftery, A., 1994. Approximate bayesian inference with the weighted likelihood bootstrap. Journal of the Royal Statistical Association Series B 56, 3-48 (with discussion and reply by the authors).

Neyman, J., Scott, E., 1948. Consistent estimation from partially consistent observations. Econometrica $16,1-32$.

Nguyen, P. V., 2005. Distribution dynamics of $\mathrm{CO}_{2}$ emissions. Environmental and Resource Economics 32, $495-508$.

Nickell, S., 1981. Biases in dynamic models with fixed effects. Econometrica 49, 1417-1426.

Nourry, M., 2009. Re-examining the empirical evidence for stochastic convergence of two air pollutants with a pair-wise approach. Environment and Resource Economics 44, 555-570.

Ordás Criado, C., Grether, J.-M., 2011. Convergence in per capita $\mathrm{CO}_{2}$ emissions: A robust distributional approach. Resource and Energy Economics 33, 637-665.

Ordás Criado, C., Valente, S., Stengos, T., 2011. Growth and pollution convergence: Theroy and evidence. Journal of Environmental Economics and Management 62, 199-214.

Panopoulou, E., Pantelidis, T., 2009. Club convergence in carbon dioxide emissions. Environmental and Resource Economicss 44, 47-70.

Pettersson, F., Maddison, D., Acar, S., Söderholm, P., 2014. Convergence of carbon dioxide emissions: A review of the literature. International Review of Environmental and Resource Economics 7, 141-178. 
Phillips, P., Sul, D., 2007a. Some empirics on economic growth under heterogenous technology. Journal of Macroeconomics 29, 455-469.

Phillips, P., Sul, D., 2007b. Transition modelling and econometric convergence tests. Econometrica 75 (6), 1771-1855.

Quah, D., 1993. Empirical cross-section dynamics in economic growth. European Economic Review 37 (23), 426-434.

Quah, D., 1996. Empirics for economic growth and convergence. European Economic Review 40, 13531375 .

Ravallion, M., 2003. Inequality convergence. Economics Letters 80, 351-356.

Romero-Ávila, D., 2008. Convergence in carbon dioxide emissions among industrialised countries revisited. Energy Economics 30, 2265-2282.

Rossi, P.E., A. G. M. R., 2005. Bayesian Statistics and Marketing. Wiley Series in Probability and Statistics. John Wiley and Sons, Chichester.

Rubin, D., 1981. The bayesian bootstrap. Annals of Statistics 9, 130-134.

Sala-i-Martin, X., 1996. Regional cohesion: Evidence and theories of regional growth and convergence. European Economic Review 40 (6), 1325-1352.

Salois, M., Balcombe, K., 2015. A generalized bayesian instrumental variable approach under student $t$-distributed errors with application. The Manchester School 83, 499-522.

Schmalensee, R., Stoker, T., Judson, R., 1998. World carbon dioxide emissions: 1950-2050. The Review of Economics and Statistics 80 (1), 15-27.

Solow, R., 1956. A contribution to the theory of economic growth. The Quarterly Journal of Economics $70(1), 65-94$.

Spiegelhalter, David J.; Best, N. G. C. B. P. v. d. L. A., 2002. Bayesian measures of model complexity and fit (with discussion). Journal of the Royal Statistical Society, Series B 64, 583-639.

Stern, D., 2004. The rise and fall of the environmental Kuznets Curve. World Development 32, 1419-1439.

Stern, D., 2017. The environmental Kuznets Curve after 25 years. Journal of Bioeconomics, 1-22.

Strazicich, M. C., List, J. A., 2003. Are $\mathrm{CO}_{2}$ emission levels converging among industrial countries? Environmental and Resource Economics 24, 263-271.

Wang, Y., Zhang, P., Huang, D., Cai, C., 2014. Convergence behavior of carbon dioxide emissions in China. Economic Modelling 43, $75-80$.

West, M., 1987. On scale mixtures of normal distributions. Biometrika 74, 646-648.

Westerlund, J., Basher, S. A., 2008. Testing for convergence in carbon dioxide emissions using a century of panel data. Environmental and Resource Economics 40, 109-120.

Wu, J., Wu, Y., Guo, X., Cheong, T. S., 2016. Convergence of carbon dioxide emissions in Chinese cities: A continuous dynamic distribution approach. Energy Policy 91, 207-219. 
Yavuz, N., Yilanci, V., 2013. Convergence in per capita carbon dioxide emissions among G7 countries: A TAR panel root approach. Environment and Resource Economics 54, 283-291.

Young, A., Higgins, M., Levy, D., 2008. Sigma convergence vs beta convergence: Evidence from U.S. country-level data. Journal of Money, Credit and Banking 40 (5), 1083-1093.

Zhao, X., Burnett, W., Lacombe, D., 2015. Province-level convergence of China's carbon dioxide emissions. Applied Energy 150, 286-295. 